\title{
Assessment of variational multiscale models for the large eddy simulation of turbulent incompressible flows
}

\author{
Oriol Colomés ${ }^{\mathrm{a}}$, Santiago Badia ${ }^{\mathrm{c}, \mathrm{a}}$, Ramon Codina ${ }^{\mathrm{c}, \mathrm{b}}$, Javier Principe ${ }^{\mathrm{d}, \mathrm{a}, *}$ \\ ${ }^{a}$ Centre Internacional de Mètodes Numèrics en Enginyeria (CIMNE), Parc Mediterrani de la Tecnologia, UPC, Esteve \\ Terradas 5, 08860 Castelldefels, Spain. \\ ${ }^{b}$ Centre Internacional de Mètodes Numèrics en Enginyeria (CIMNE), Jordi Girona 1-3, Campus Nord UPC, Edifici \\ C1, 08034 Barcelona, Spain. \\ ${ }^{c}$ Universitat Politècnica de Catalunya Jordi Girona 1-3, Campus Nord UPC, Edifici C1, 08034 Barcelona, Spain. \\ ${ }^{d}$ Universitat Politècnica de Catalunya, Urgell 187, 08036 Barcelona, Spain.
}

\begin{abstract}
In this work we study the performance of some variational multiscale models (VMS) in the large eddy simulation (LES) of turbulent flows. We consider VMS models obtained by different subgrid scale approximations which include either static or dynamic subscales, linear or nonlinear multiscale splitting and different choices of the subscale space. After a brief review of these models, we discuss some implementation aspects particularly relevant to the simulation of turbulent flows, namely the use of a skew symmetric form of the convective term and the computation of projections when orthogonal subscales are used. We analyze the energy conservation (and numerical dissipation) of the alternative VMS formulations, which is numerically evaluated. In the numerical study, we have considered three well known problems: the decay of homogeneous isotropic turbulence, the Taylor-Green vortex problem and the turbulent flow in a channel. We compare the results obtained using the different VMS models and against a classical LES scheme based on filtering and the Smagorinsky closure. Altogether, our results show the tremendous potential of VMS for the numerical simulation of turbulence. Further, we study the sensitivity of VMS to the algorithmic constants and analyze the behavior in the small time step limit. We have also carried out a computational cost comparison of the different formulations. Out of these results, we can state that the numerical results obtained with the different VMS formulations (as far as they converge) are quite similar. However, some choices are prone to instabilities and the results obtained in terms of computational cost are certainly different. The dynamic orthogonal subscales model turns out to be best in terms of efficiency and robustness.
\end{abstract}

Keywords: turbulence, large eddy simulation, stabilization, variational multiscale

\section{Introduction}

LES techniques for the numerical simulation of turbulent flows [54] are based on a scale separation that permits to reduce the computational cost with respect to direct numerical simulation (DNS). Such scale separation is traditionally achieved by filtering the original Navier-Stokes equations, which leads to an extra forcing term defined by a physical (functional or structural) model. This widely used approach is usually referred to as explicit LES [54].

By contrast, implicit LES techniques (ILES) rely on purely numerical artifacts without any modification of the continuous problem. This approach was seldom followed, the MILES (Monotone Integrated LES) approach [12, 26, 30, 49] being the main exception, until the VMS method was introduced [36, 37] and subsequently proposed as a LES method (see below). ILES techniques are usually considered to be based on the addition of purely dissipative numerical terms, see [54, Section 5.3.4]. It is worth to

\footnotetext{
${ }^{*}$ Corresponding author

Email addresses: ocolomes@cimne.upc.edu (Oriol Colomés), sbadia@cimne.upc.edu (Santiago Badia), ramon.codina@upc.edu (Ramon Codina), principe@cimne.upc.edu (Javier Principe)
} 
emphasize that this is not the case of some particular VMS models, as it is shown in [52] and discussed below.

VMS was introduced in $[36,37]$ as a framework for the development of stabilization techniques, which aim to overcome numerical difficulties encountered when using the standard Galerkin method. On the one hand, the velocity and pressure finite element (FE) spaces need to satisfy the inf-sup compatibility condition that guarantees pressure stability and precludes the use of equal order interpolation. Mixed methods satisfying this condition can be used and their finite volume counterpart, based on staggered grids, are common in the LES community. Stabilization techniques that permit the use of equal order interpolation were proposed, e.g., in $[24,38]$, and finally recast into the VMS framework. On the other hand, global nonphysical oscillations appear in the convection dominated regime, when the mesh is not fine enough, that is, for high mesh Reynolds number flows. The only way to overcome this problem is through the addition of some form of dissipation which was recognized in the early development of stabilized methods [14]. Let us note that the common practice in the LES community is to rely on the explicit extra term introduced by the physical model using high order approximations of the convective term.

The first attempts to perform LES using VMS models were presented in [39, 40, 44]. The VMS models used in these works divide resolved scales into large and small introducing a Smagorinsky-type dissipative term acting on the small scales only. As a result, an important fraction of the degrees of freedom are used for the small resolved scales whereas consistency is retained in the large resolved scales only. ILES using a VMS approach with a resolved and a modeled subgrid scale (the setting that permits to recover stabilized formulations) was suggested in [19] and performed in [16, 10]. Excellent

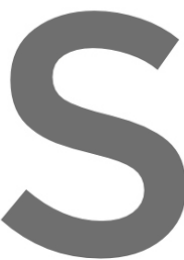
results were first prese Compared to classical with inhomogeneous n in the $\mathrm{FE}$ equations up th consistency error of or Scale separation is

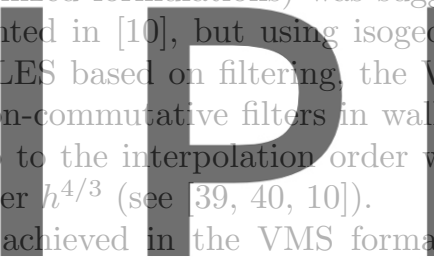

unknown is split into a resolvable FE component and a subgrid or subscale component. The action Register for the subscales ont the FE scales can be approximated in different ways, leading to different VMS

space was advocated. The possibility of considering the subscales time dependent and to keep the VMS decomposition in all the nonlinear terms was studied [19,22] and important improvements have been observed when using these models for the simulation of laminar flows [22, 2].

In this work we assess these VMS models in the numerical simulation of turbulent flows. We present a detailed numerical experimentation for three well known problems: the decay of homogeneous isotropic turbulence (DHIT), the Taylor-Green vortex (TGV) and the turbulent flow in a channel (TCF). Thus, both unbounded and bounded flows are considered.

We refer to the original references for a comprehensive treatment of their assumptions and their numerical analysis. Our intention here is to compare the different VMS schemes in terms of quality of the results and computational cost and discuss some implementation aspects that we find particularly relevant for the simulation of turbulent flows. The first of them is the treatment of the convective term. As it is well-known, the numerical analysis requires a skew-symmetric form of this term in order to avoid any positive contribution to the energy estimates that cannot be properly controlled. We show numerically that such a contribution actually appears if a non skew-symmetric form is used. Another point that deserves attention is the use of consistent mass matrices to perform projections when orthogonal subscales are considered. Even though it is cheaper to use lumped mass matrices, only the use of consistent matrices guarantees exact $L^{2}$ orthogonality. An alternative is the use of Scott-Zhang projections recently proposed in [4], although we do not consider this approach here.

We also discuss the influence of the algorithmic constants in the stabilization parameters in the

\footnotetext{
${ }^{1}$ It is worth to point out that both problems (convection instability and compatibility conditions) are also present in the linear Oseen problem. One of the weakness of an explicit LES approach without a numerical dissipation term is that convection is stabilized by a term that comes from the physical model of the nonlinear Navier Stokes equations and such a term is not present when the linear Oseen problem is considered.
} 
numerical results. In particular, we show that the choice of the second stabilization parameter (the one multiplying the div-div term) has an important influence on the numerical results while it is not essential for stability and convergence of the methods. We further analyze the behavior of the VMS formulation as the time step size is reduced. These two facts are actually related by the way the stabilization parameters are usually defined (see $[27,34]$ ).

Finally, we compare the results obtained using VMS models against those obtained using classical LES based on filtering and the Smagorinsky closure. We do so using the Galerkin approximation of the Navier Stokes equations with a Taylor-Hood $Q 2 / Q 1$ interpolation which satisfies the inf-sup condition, relying on the Smagorinsky term to stabilize convection as it is usually done in the LES community.

The article is organized as follows. In Section 2 we present the VMS formulation, how to compute truly orthogonal subscales and the different models we aim at analyzing, whereas in Section 3 we discuss energy conservation statements and how they are influenced by the choice of the VMS method and the definition of the convective term. Sections 5, 6,7 are devoted to the numerical approximation of the DHIT, the TGV and the TCF problems. Sections 8 and 9 discuss the effect of the algorithmic constants on the results and the behavior of the different schemes in the small time step limit. Some remarks close the article in Section 10.

\section{Formulation}

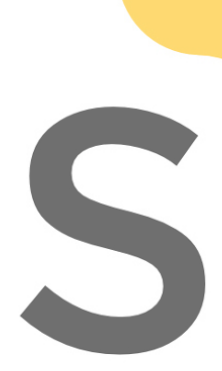

2.1. Navier-Stokes problem

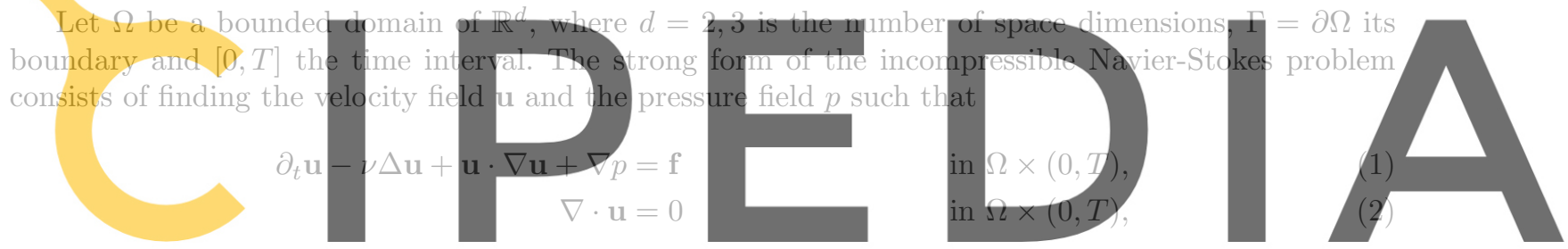

with $\mathrm{f}$ the force vector and $\nu$ the kinematic viscosity. Hereinafter bold characters denote vectors and

Equations (1)-(2) have to be supplied with appropriate boundary and initial conditions. The boundary $\Gamma$ is divided into the Dirichlet $\left(\Gamma_{D}\right)$ and the Neumann $\left(\Gamma_{N}\right)$ parts such that $\Gamma_{D} \cup \Gamma_{N}=\Gamma_{\text {and }}$ $\Gamma_{D} \cap \Gamma_{N}=\varnothing$. Then, the boundary and initial conditions can be written as

$$
\begin{aligned}
\mathbf{u} & =\mathbf{u}_{g} & & \text { on } \Gamma_{D} \times(0, T], \\
\left(-p \mathbf{I}+\nu\left(\nabla \mathbf{u}+\nabla \mathbf{u}^{T}\right)\right) \cdot \mathbf{n} & =\mathbf{t}_{N} & & \text { on } \Gamma_{N} \times(0, T], \\
\mathbf{u}(\mathbf{x}, 0) & =\mathbf{u}_{0}(\mathbf{x}) & & \text { in } \Omega \times\{0\},
\end{aligned}
$$

$\mathbf{n}$ being the unit outward vector normal to $\Gamma$. To simplify the exposition, we will consider $\mathbf{u}_{g}=\mathbf{0}$ and $\Gamma_{D}=\Gamma$ in what follows.

In order to derive the weak form of the problem (1)-(5) we define some notation that will be used hereafter. We denote by $L^{p}(\Omega), 1 \leq p<\infty$, the spaces of functions such that their $p$-th power is absolutely integrable in $\Omega$. For the case in which $p=2$, we have a Hilbert space with scalar product

$$
(u, v)_{\Omega} \equiv(u, v):=\int_{\Omega} u(\mathbf{x}) v(\mathbf{x}) d \Omega
$$

and induced norm $\|u\|_{L^{2}(\Omega)} \equiv\|u\|=(u, u)^{1 / 2}$. Abusing of the notation, the same symbol as in (6) will be used for the integral of the product of two functions, even if these are not in $L^{2}(\Omega)$, and both for scalar and vector fields. The space of functions whose distributional derivatives up to order $m$ are in the $L^{2}(\Omega)$ space are denoted by $H^{m}(\Omega)$. We will focus on the case of $m=1$, which is also a Hilbert space. $H_{0}^{1}(\Omega)$ is the set of functions in $H^{1}(\Omega)$ that have zero trace on $\Gamma_{D}$. Furthermore, we denote by $H^{-1}(\Omega)$ the topological dual of $H_{0}^{1}(\Omega)$ and by $\langle\cdot, \cdot\rangle$ the duality pairing between $H^{-1}(\Omega)$ and $H_{0}^{1}(\Omega)$. Given a Banach space $X, L^{p}(0, T ; X)$ is the space of time dependent functions such that their $X$-norm is in $L^{p}(0, T)$. 
The weak form of the incompressible Navier-Stokes problem (1)-(5) consists, e.g., in finding $[\mathbf{u}, p] \in$ $L^{2}\left(0, T ; \mathcal{V}_{0}\right) \times \mathcal{D}^{\prime}\left(0, T ; \mathcal{Q}_{0}\right)$ (distributions in time with values in $\left.\mathcal{Q}_{0}\right)$ such that

$$
\left(\partial_{t} \mathbf{u}, \mathbf{v}\right)+B(\mathbf{u} ;[\mathbf{u}, p],[\mathbf{v}, q])=\langle\mathbf{f}, \mathbf{v}\rangle \quad \forall \mathbf{v} \in \mathcal{V}_{0}, \quad \forall q \in \mathcal{Q}_{0},
$$

satisfying the initial condition (5) in a weak sense. Here $\mathcal{V}_{0}:=H_{0}^{1}(\Omega)^{d}, \mathcal{Q}_{0}:=L^{2}(\Omega) / \mathbb{R}$ and the form $B(\mathbf{a} ;[\mathbf{u}, p],(\mathbf{v}, q))$ is defined as

$$
B(\mathbf{a} ;[\mathbf{u}, p],[\mathbf{v}, q]):=\nu(\nabla \mathbf{u}, \nabla \mathbf{v})+b(\mathbf{a}, \mathbf{u}, \mathbf{v})-(p, \nabla \cdot \mathbf{v})+(q, \nabla \cdot \mathbf{u})
$$

where the trilinear weak form of the convective term $b(\mathbf{u}, \mathbf{v}, \mathbf{w})$ can be written in the following three equivalent ways

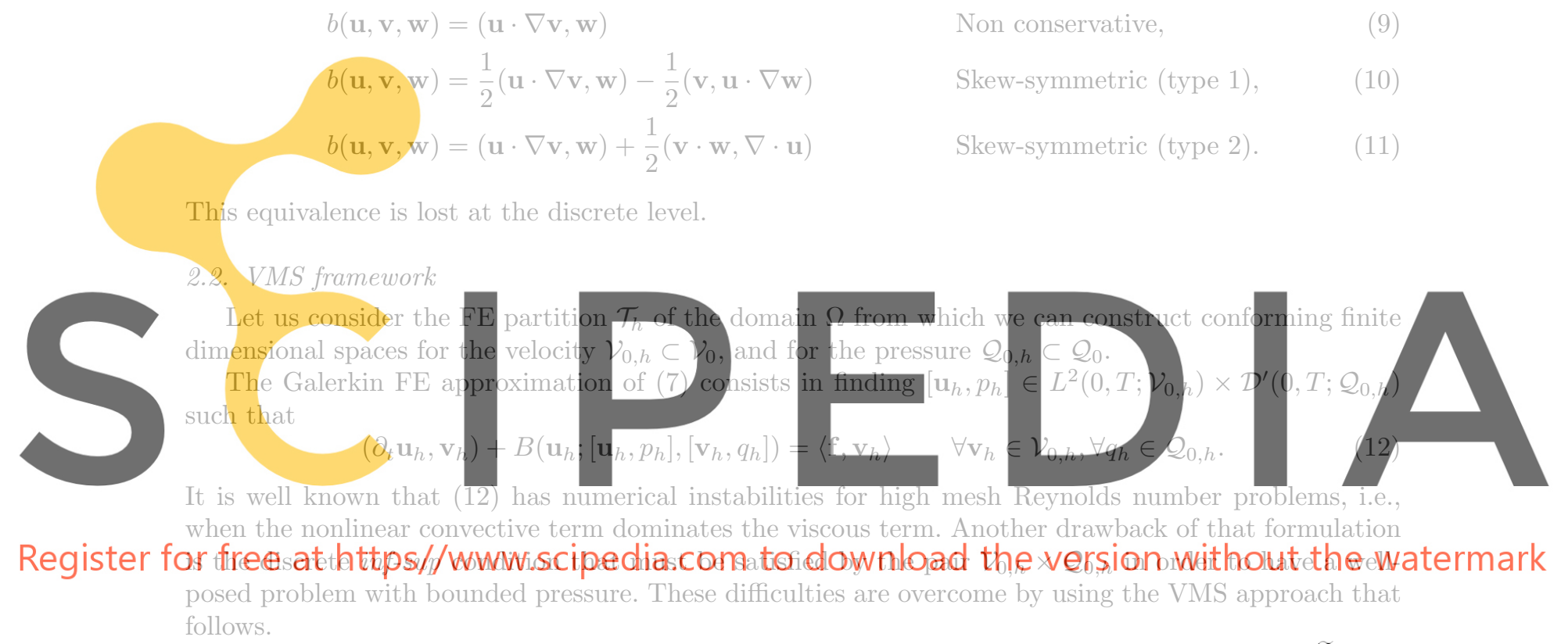

Let us consider a two-scale decomposition of spaces $\mathcal{V}_{0}$ and $\mathcal{Q}_{0}$ such that $\mathcal{V}_{0}=\mathcal{V}_{0, h} \oplus \widetilde{\mathcal{V}}_{0}$ and $\mathcal{Q}=\mathcal{Q}_{0, h} \oplus \widetilde{\mathcal{Q}}_{0}$, where $\widetilde{\mathcal{V}}_{0}$ and $\widetilde{\mathcal{Q}}_{0}$ are infinite-dimensional spaces that complete the FE spaces in $\mathcal{V}_{0}$ and $\mathcal{Q}_{0}$, respectively. Hereinafter the subscript $(\cdot)_{h}$ will denote the FE component and the tilde $\widetilde{(\cdot)}$ the subgrid component. Applying the two-scale decomposition to (7) we obtain a discrete problem

$$
\left(\partial_{t} \mathbf{u}_{h}, \mathbf{v}_{h}\right)+\left(\partial_{t} \tilde{\mathbf{u}}, \mathbf{v}_{h}\right)+B\left(\mathbf{a} ;\left[\mathbf{u}_{h}, p_{h}\right],\left[\mathbf{v}_{h}, q_{h}\right]\right)+\left(\tilde{\mathbf{u}}, \mathcal{L}_{\mathbf{a}}^{*}\left(\mathbf{v}_{h}, q_{h}\right)\right)_{h}-\left(\tilde{p}, \nabla \cdot \mathbf{v}_{h}\right)=\left\langle\mathbf{f}, \mathbf{v}_{h}\right\rangle
$$

where $(\cdot, \cdot)_{h}=\sum_{K \in \mathcal{T}_{h}}(\cdot, \cdot)_{K}$ is the sum of scalar products (6) over each element $K$ of the partition $\mathcal{T}_{h}$, and

$$
\mathcal{L}_{\mathbf{a}}^{*}\left(\mathbf{v}_{h}, q_{h}\right):=-\nu \nabla^{2} \mathbf{v}_{h}-\mathbf{a} \cdot \nabla \mathbf{v}_{h}-\nabla q_{h}
$$

is the formal of the adjoint operator of the momentum equation. The term involving the adjoint operator comes from an elementwise integration by parts of the terms involving the subscales, in which the boundary terms $\left(\mathbf{v}_{h}, \nu \mathbf{n} \cdot \nabla \tilde{\mathbf{u}}\right)_{\partial h}$ and $\left(q_{h}, \mathbf{n} \cdot \tilde{\mathbf{u}}\right)_{\partial h}$ have been neglected (here $\partial h$ means the sum over all elements of the integral on the boundary of each element). It also involves the approximation $b\left(\mathbf{a}, \tilde{\mathbf{u}}, \mathbf{u}_{h}\right) \approx-\left(\tilde{\mathbf{u}}, \mathbf{a} \cdot \nabla \mathbf{v}_{h}\right)$ which implies neglecting $\left(\mathbf{v}_{h}, \mathbf{n} \cdot \mathbf{a} \tilde{\mathbf{u}}\right)_{\partial h}$ and $\left(\tilde{\mathbf{u}}, \nabla \cdot \mathbf{a v}_{h}\right)$. These approximations are discussed in [22] together with the choice of a which defines the type of scale splitting (linear or nonlinear), also discussed below.

The discrete problem depends on $\tilde{\mathbf{u}} \in \widetilde{\mathcal{V}}_{0}$ and on $\tilde{p} \in \widetilde{\mathcal{Q}}_{0}, \widetilde{\mathcal{V}}_{0}$ and $\widetilde{\mathcal{Q}}_{0}$ being infinite-dimensional. Therefore, the equations for $\tilde{\mathbf{u}}$ and $\tilde{p}$ obtained after applying the two-scale decomposition cannot be directly solved, but some modeling steps are needed to obtain a feasible method. Considering the subscale as a time-dependent variable of the problem (see below) and approximating the Navier-Stokes operator 
by two stabilization parameters $\tau_{m}^{-1}$ and $\tau_{c}^{-1}$ (see for example [22]), the fine scale problem can be written as

$$
\begin{aligned}
\partial_{t} \tilde{\mathbf{u}}+\tau_{m}^{-1} \tilde{\mathbf{u}} & =\mathcal{P}\left(\mathbf{R}_{u}\right), \\
\tau_{c}^{-1} \tilde{p} & =\mathcal{P}\left(R_{p}\right) .
\end{aligned}
$$

In (15)-(16) $\mathcal{P}$ denotes the projection onto the space of subscales, which is discussed below. In turn, the vector $\mathbf{R}$ is the residual of the Navier-Stokes equations (1)-(2), defined as $\mathbf{R}=\left[\mathbf{R}_{u}, R_{p}\right]^{T}$, with

$$
\begin{aligned}
& \mathbf{R}_{u}=\mathbf{f}-\partial_{t} \mathbf{u}_{h}-\mathcal{L}_{\mathbf{a}}\left(\mathbf{u}_{h}, p_{h}\right), \\
& R_{p}=-\nabla \cdot \mathbf{u}_{h} .
\end{aligned}
$$

where

$$
\mathcal{L}_{\mathbf{a}}\left(\mathbf{v}_{h}, q_{h}\right):=-\nu \nabla^{2} \mathbf{v}_{h}+\mathbf{a} \cdot \nabla \mathbf{v}_{h}+\nabla q_{h}
$$

Finally, the expressions of the stabilization parameter $\tau_{m}$ is

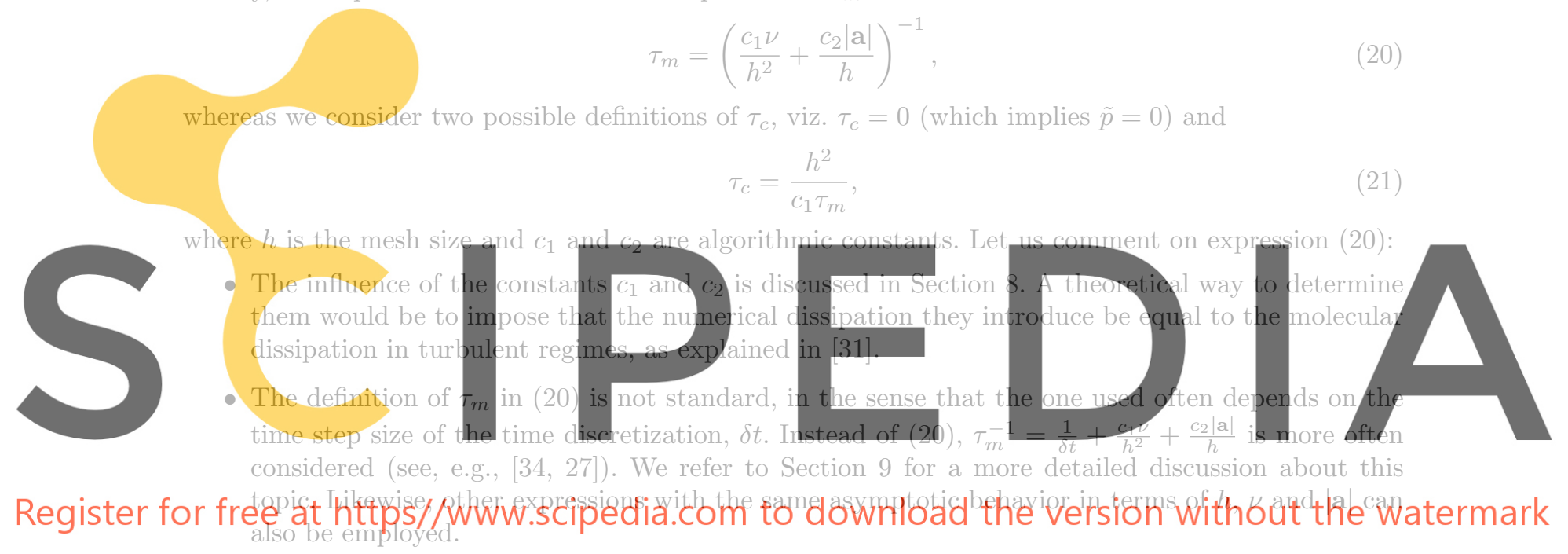

- Expression (20) corresponds to linear isotropic elements. If elements of oder $p$ are used ( $p$ is not the pressure, here), $c_{1}$ must be replaced by $c_{1} p^{4}$ and $c_{2}$ by $c_{2} p$. For anisotropic elements, the definition of $h$ within each element is not obvious. A possibility is explained in [51].

In the following three sections we discuss the particular ingredients of our VMS models. A different summary can also be found in [21], together with some numerical experiments.

\subsubsection{The dynamics of the subscales}

Stabilized formulations were originally developed for steady convection-diffusion [14] and Stokes $[24,38]$ problems. As the numerical instabilities have a spatial nature, the time dependency of the subscales was not considered, and the standard choice $[39,40,10]$ was to take

$$
\tilde{\mathbf{u}}=\tau_{m} \mathcal{P}\left(\mathbf{R}_{u}\right)
$$

that is, to neglect the temporal derivative of the subscales in (15). In this case, the subscales are called quasi-static in what follows.

The subscale as a time dependent variable of the problem was introduced in [19, 22]. It gives rise to important properties like commutativity of space and time discretization, stability without restrictions on the time step size $[22,5]$ and, combined with orthogonal subscales, to convergence towards weak solutions of the Navier-Stokes equations [7] and the possibility of predicting backscatter [21, 52].

Equation (15) can be analytically integrated to give

$$
\tilde{\mathbf{u}}\left(t^{*}\right)=\tilde{\mathbf{u}}(0)+\mu^{-1}\left(t^{*}\right) \int_{0}^{t^{*}} \mu(t) \mathcal{P} \mathbf{R}_{u} \mathrm{~d} t, \quad \mu(s)=\exp \int_{0}^{s} \tau^{-1}(t) \mathrm{d} t,
$$

where it is explicitly seen that the subscale is a function of the residual but also of the flow history. In practice this integration is performed numerically, as described below. 


\subsection{2. (Non)linear scale splitting}

The original VMS formulation $[36,37]$ was developed having linear problems in mind and its extension to the Navier-Stokes equations was implicitly based on a "linearization", fixing the advection velocity and applying the multiscale splitting to the rest of the terms. A nonlinear scale splitting was used in $[39,40]$ together with an explicit resolution of the small scales in which a Smagorinsky damping was introduced. A nonlinear scale splitting with modeled subscales was used in [19, 10] and in [22], where it was shown that it leads to global conservation of momentum. We therefore consider both options

$$
\begin{array}{ll}
\mathbf{a}=\mathbf{u}_{h} & \text { for linear subscales, } \\
\mathbf{a}=\mathbf{u}_{h}+\tilde{\mathbf{u}} & \text { for nonlinear subscales. }
\end{array}
$$

Remark 2.1. When we use the nonlinear definition for the advection velocity, $\mathbf{a}=\mathbf{u}_{h}+\tilde{\mathbf{u}}$, the skewsymmetric term type $2(11)$ in the FE equation (13) reads:

$$
b\left(\mathbf{a}, \mathbf{u}_{h}, \mathbf{v}_{h}\right)=\left(\left(\mathbf{u}_{h}+\tilde{\mathbf{u}}\right) \cdot \nabla \mathbf{u}_{h}, \mathbf{v}_{h}\right)+\frac{1}{2}\left(\mathbf{u}_{h} \cdot \mathbf{v}_{h}, \nabla \cdot \mathbf{u}_{h}\right)+\frac{1}{2}\left(\mathbf{u}_{h} \cdot \mathbf{v}_{h}, \nabla \cdot \tilde{\mathbf{u}}\right) .
$$

The last term is not well-defined, since it includes derivatives of the discontinuous subscale ũ. One possibility is to neglect it (as previously done with other similar terms when arriving to (13)), which implies
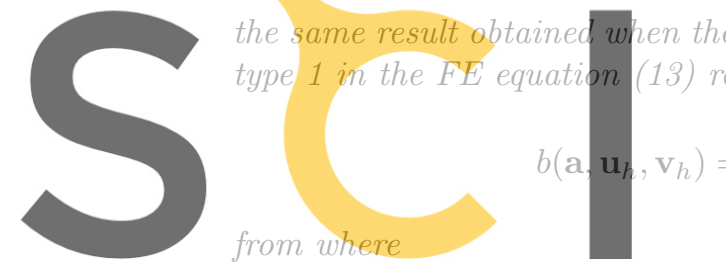

$$
b\left(\mathrm{a}, \mathrm{u}_{h}, \mathrm{u}_{h}\right)=-\frac{1}{2}\left(\left|\mathbf{u}_{h}\right|^{2}, \nabla \cdot \tilde{\mathbf{u}}\right),
$$

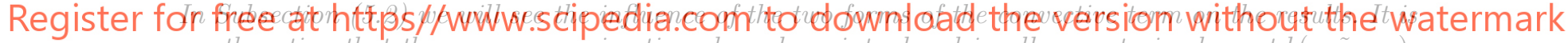 worth noting that the same approximations have been introduced in all cases to implement $b\left(\mathbf{a}, \mathbf{u}_{1}, \mathbf{u}_{h}\right)$, but these approximations are taken into account in the (usual) energy estimates of Section 3.}

Remark 2.2. At the continuous level, the different expressions of the convective term are also equivalent to the so called conservation form

$$
b(\mathbf{u}, \mathbf{v}, \mathbf{w})=-(\mathbf{u} \otimes \mathbf{v}, \nabla \mathbf{w}) .
$$

In the discrete problem, the nonlinear scale splitting leads to the following terms in the momentum equation:

$$
b\left(\mathbf{a}, \mathbf{u}_{h}+\tilde{\mathbf{u}}, \mathbf{v}_{h}\right)=-\left(\mathbf{u}_{h} \otimes \mathbf{u}_{h}, \nabla \mathbf{v}_{h}\right)-\left(\mathbf{u}_{h} \otimes \tilde{\mathbf{u}}, \nabla \mathbf{v}_{h}\right)-\left(\tilde{\mathbf{u}} \otimes \mathbf{u}_{h}, \nabla \mathbf{v}_{h}\right)-\left(\tilde{\mathbf{u}} \otimes \tilde{\mathbf{u}}, \nabla \mathbf{v}_{h}\right) .
$$

Even if this is not exactly what we get using the non-conservative or skew-symmetric forms because of the approximation error, this allows us to interpret the different contributions arising from the nonlinear scale splitting. As it is explained in [21], from (30) we can identify the contributions from the cross stresses, the Reynolds stresses and the subgrid scale tensor.

\subsubsection{The space for the subscales}

The selection of the space for the approximation of the subscales determines the projection $\mathcal{P}$ appearing in the right-hand side of (15) and (16). The first option, already considered in [39, 40,10] and named Algebraic Subgrid Scale (ASGS) in [18] is to take the subscales in the space of the residuals, that is,

$$
\mathcal{P}:=\mathbf{I} \text {. }
$$

Another possibility introduced in [18] is to consider the space of the subscales orthogonal to the FE space. The main motivation of the method is that a stability estimate for the projection onto the FE 
space of the pressure and/or the convective terms can already be obtained in the standard Galerkin method and therefore the only "missing" part is the orthogonal one. The Orthogonal Subscales (OSS) method is then characterized by the following projection definition:

$$
\mathcal{P}:=\Pi_{h}^{\perp}=\mathbf{I}-\Pi_{h},
$$

where $\Pi_{h}$ is the projection onto the FE space. With this choice, the residual of the momentum equation does not depend on $\partial_{t} \mathbf{u}_{h}$. Likewise, $\mathcal{P}(\mathbf{f})$ in this case is only well defined for $\mathbf{f} \in L^{2}(\Omega)^{d}$. In the case of minimum regularity, $\mathbf{f} \in H^{-1}(\Omega)^{d}$, this term can be simply neglected without upsetting the accuracy of the method.

In fact, with this choice, the orthogonality between the space of subscales and the FE space is only guaranteed when the stabilization parameters are constant. If this is not the case, the method is still optimally convergent [20] but this property is lost. In order to have truly orthogonal subscales, which guarantees a proper separation of the FE and the subgrid scale kinetic energies (see below and Section 3) a slight modification of the projection $\Pi_{h}$ is needed (see [20]). We will use two different weighted projections: one for the velocity subscales $\left(\Pi_{m}\right)$ in $(15)$ and another for the pressure subscales $\left(\Pi_{c}\right)$ in (16). We define the weighted projections $\Pi_{m}$ and $\Pi_{c}$ such that given any vector $\mathbf{w} \in \mathcal{V}_{0}$ and any scalar $r \in \mathcal{Q}_{0}$ we have

$$
\begin{array}{ll}
\left(\tau_{m} \Pi_{m}(\mathbf{w}), \mathbf{v}_{h}\right)=\left(\tau_{m} \mathbf{w}, \mathbf{v}_{h}\right) & \forall \mathbf{v}_{h} \in \mathcal{V}_{0, h}, \\
\left(\tau_{c} \Pi_{c}(r), q_{h}\right)=\left(\tau_{c} r, q_{h}\right) & \forall q_{h} \in \mathcal{Q}_{0, h} .
\end{array}
$$

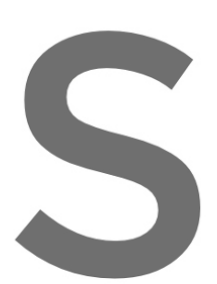

These definitions guar: subscales, that is, negl temporal derivative of

However, if the dyn conveniently modified of the weight depends
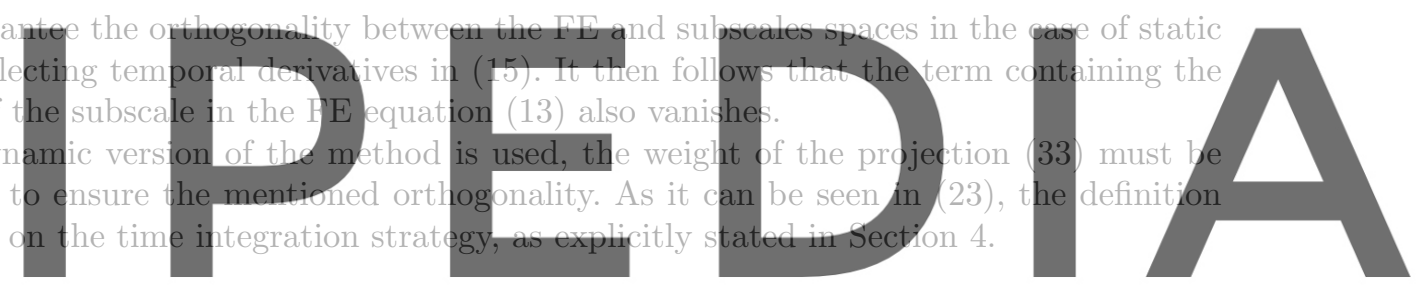

3. Energy balance statements

Register for free at https//www.scipedia.com to download the version without the watermark

In this section we revisit global energy conservation statements of the method. As shown in [52],

similar statements can be obtained locally (in a volume $\omega \subset \Omega$ ).

Taking $\mathbf{v}_{h}=\mathbf{u}_{h}$ and $q_{h}=p_{h}$ in (13) we have the energy balance on the $\mathrm{F} \mathbf{E}$ component

$$
\begin{aligned}
\underbrace{\frac{1}{2} d_{t}\left\|\mathbf{u}_{h}\right\|^{2}}_{I} & +\underbrace{\nu\left\|\nabla \mathbf{u}_{h}\right\|^{2}}_{I I}+\underbrace{b\left(\mathbf{a}, \mathbf{u}_{h}, \mathbf{u}_{h}\right)}_{I I I} \\
& +\underbrace{\left(\partial_{t} \tilde{\mathbf{u}}, \mathbf{u}_{h}\right)+\left(\tilde{\mathbf{u}}, \mathcal{L}_{\mathbf{a}}^{*}\left(\mathbf{u}_{h}, p_{h}\right)\right)_{h}-\left(\tilde{p}, \nabla \cdot \mathbf{u}_{h}\right)}_{I V}=\underbrace{\left\langle\mathbf{f}, \mathbf{u}_{h}\right\rangle}_{V},
\end{aligned}
$$

In equation (35) we group the terms as
I) FE kinetic energy variation:
$\frac{1}{2} d_{t}\left\|\mathbf{u}_{h}\right\|^{2}$
II) $\mathrm{FE}$ viscous dissipation:
III) FE convective term:
$\nu\left\|\nabla \mathbf{u}_{h}\right\|^{2}$
IV) FE to SGS energy transfer:
V) FE component of external power:
$b\left(\mathbf{a}, \mathbf{u}_{h}, \mathbf{u}_{h}\right)$
$\varepsilon_{h}=\left(\partial_{t} \tilde{\mathbf{u}}, \mathbf{u}_{h}\right)+\left(\tilde{\mathbf{u}}, \mathcal{L}_{\mathbf{a}}^{*}\left(\mathbf{u}_{h}, p_{h}\right)\right)_{h}-\left(\tilde{p}, \nabla \cdot \mathbf{u}_{h}\right)$
$\left\langle\mathbf{f}, \mathbf{u}_{h}\right\rangle$

Multiplying (15) by $\tilde{\mathbf{u}}$ and (16) by $\tilde{p}$, integrating over the domain and decomposing the residual of the momentum equation as $\mathbf{R}_{u}=\mathbf{f}-\partial_{t} \mathbf{u}_{h}-\mathcal{L}_{\mathbf{a}}\left(\mathbf{u}_{h}, p_{h}\right)$, we obtain the global energy balance on the fine scale

$$
\begin{aligned}
\underbrace{\frac{1}{2} d_{t}\|\tilde{\mathbf{u}}\|^{2}}_{I} & +\underbrace{\tau_{m}^{-1}\|\tilde{\mathbf{u}}\|^{2}}_{I I}+\underbrace{\tau_{c}^{-1}\|\tilde{p}\|^{2}}_{I I I} \\
& +\underbrace{\left(\mathcal{P}\left(\partial_{t} \mathbf{u}_{h}\right), \tilde{\mathbf{u}}\right)+\left(\mathcal{P}\left(\mathcal{L}_{\mathbf{a}}\left(\mathbf{u}_{h}, p_{h}\right)\right), \tilde{\mathbf{u}}\right)_{h}+\left(\mathcal{P}\left(\nabla \cdot \mathbf{u}_{h}\right), \tilde{p}\right)}_{I V}=\underbrace{(\mathcal{P}(\mathbf{f}), \tilde{\mathbf{u}})}_{V} .
\end{aligned}
$$


We group the terms in (36) as
I) SGS kinetic energy variation:
$\frac{1}{2} d_{t}\|\tilde{\mathbf{u}}\|^{2}$
$\tau_{m}^{-1}\|\tilde{\mathbf{u}}\|^{2}$
II) SGS velocity dissipation:
$\tau_{c}^{-1}\|\tilde{p}\|^{2}$
III) SGS pressure dissipation:
$\tilde{\varepsilon}=\left(\mathcal{P}\left(\partial_{t} \mathbf{u}_{h}\right), \tilde{\mathbf{u}}\right)+\left(\tilde{\mathbf{u}}, \mathcal{P}\left(\mathcal{L}_{\mathbf{a}}\left(\mathbf{u}_{h}, p_{h}\right)\right)\right)_{h}+\left(\tilde{p}, \mathcal{P}\left(\nabla \cdot \mathbf{u}_{h}\right)\right)$
IV) SGS to FE energy transfer:
V) SGS component of external power: $(\mathcal{P}(\mathbf{f}), \tilde{\mathbf{u}})$

Finally, adding up equations (35) and (36) we obtain an equation for the total kinetic energy

$$
\begin{gathered}
\frac{1}{2} d_{t}\left\|\mathbf{u}_{h}\right\|^{2}+\frac{1}{2} d_{t}\|\tilde{\mathbf{u}}\|^{2}+\nu\left\|\nabla \mathbf{u}_{h}\right\|^{2}+b\left(\mathbf{a}, \mathbf{u}_{h}, \mathbf{u}_{h}\right)+\tau_{m}^{-1}\|\tilde{\mathbf{u}}\|^{2}+\tau_{c}^{-1}\|\tilde{p}\|^{2} \\
+\left(\partial_{t} \tilde{\mathbf{u}}, \mathbf{u}_{h}\right)+\left(\mathcal{P}\left(\partial_{t} \mathbf{u}_{h}\right), \tilde{\mathbf{u}}\right)+\left(\mathcal{P}\left(\mathcal{L}_{\mathbf{a}}\left(\mathbf{u}_{h}, p_{h}\right)\right)+\mathcal{L}_{\mathbf{a}}^{*}\left(\mathbf{u}_{h}, p_{h}\right), \tilde{\mathbf{u}}\right)_{h} \\
+\left(\mathcal{P}\left(\nabla \cdot \mathrm{u}_{h}\right)-\nabla \cdot \mathrm{u}_{h}, \tilde{p}\right)=\left\langle\mathrm{f}, \mathbf{u}_{h}\right\rangle+((\mathcal{P}(\mathbf{f}), \tilde{\mathbf{u}}) .
\end{gathered}
$$

Let us note the presence of $b\left(\mathbf{a}, \mathbf{u}_{h}, \mathbf{u}_{h}\right)$, which is zero only when the skew-symmetric type 1 form is considered. Other choices could result in a spurious positive contribution to the FE kinetic energy as it is actually observed in the DHIT problem and could result in a loss of stability, although that was not observed.

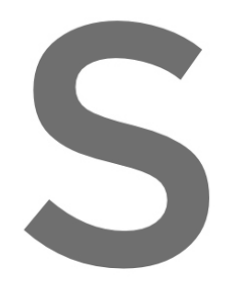

3.1. Static subscales

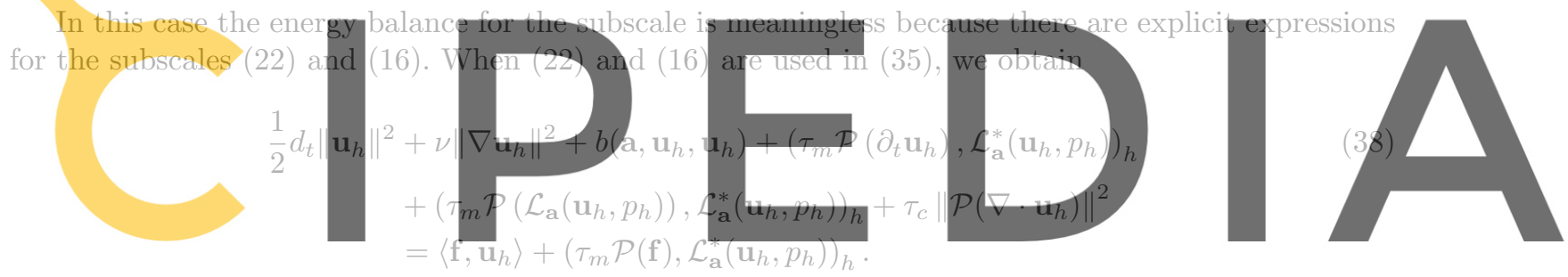

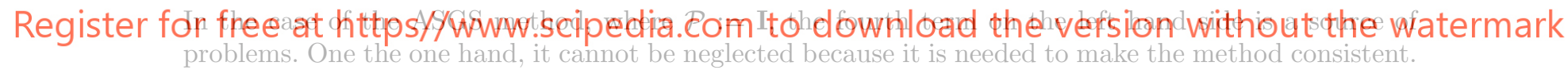

On the other hand, it can only be controlled by the dissipation of the time integration scheme and is

therefore responsible for the introduction of a restriction on the time step size. As a side problem, it is very inconvenient for an implementation if any explicit (operator splitting) time integration is chosen as it results in a nonsymmetric mass matrix. This term is not present if the OSS method is chosen using the projection $\mathcal{P}:=\mathbf{I}-\Pi_{h}$. Stability of both the fully discrete and the semidiscrete Stokes problem have been proven in [5].

The important term is the fifth one, which permits to control $\tau_{m}\left\|\mathcal{P}\left(\mathbf{a} \cdot \nabla \mathbf{u}_{h}+\nabla p_{h}\right)\right\|^{2}$; the FE part in the OSS formulation is readily controlled using inverse estimates. It therefore provides the essential numerical stability. The last term acts as a penalty on the divergence constraint, adding volumetric diffusion and provides (extra, non-essential) numerical stability.

For the OSS method, it is proved in [31] that the dissipative structure of the discrete problem has the same statistical behavior in fully developed turbulence than the continuous problem, in the sense that this dissipation has the same estimates as the molecular one. Both dissipations could be made equal by a proper choice of the stabilization parameters in (20). This, however, requires a small change in the advection velocity of this expression, which depends on an integral length of the problem. See [31] for details.

\subsection{Dynamic subscales}

In this case both the FE and subgrid components are to be considered and an estimator for both the FE and subgrid velocity can be obtained. The stability of the subgrid scale velocity can then be used to obtain a stability estimate of the FE component in a norm that includes the convective and 
pressure terms $[22,5,6] .{ }^{2}$ Therefore, the numerical dissipation of the method is actually given by the energy transfer $\varepsilon_{h}$ from the FE to the subscale component. Using (15)-(16), we get:

$$
\begin{aligned}
\varepsilon_{h} & =\left(\partial_{t} \tilde{\mathbf{u}}, \mathbf{u}_{h}\right)-\left(\tau_{m} \partial_{t} \tilde{\mathbf{u}}, \mathcal{L}_{\mathbf{a}}^{*}\left(\mathbf{u}_{h}, p_{h}\right)\right)_{h}-\left(\tau_{m} \mathcal{P}\left(\partial_{t} \mathbf{u}_{h}\right), \mathcal{L}_{\mathbf{a}}^{*}\left(\mathbf{u}_{h}, p_{h}\right)\right)_{h} \\
& -\left(\tau_{m} \mathcal{P}\left(\mathcal{L}_{\mathbf{a}}\left(\mathbf{u}_{h}, p_{h}\right)\right), \mathcal{L}_{\mathbf{a}}^{*}\left(\mathbf{u}_{h}, p_{h}\right)\right)_{h}+\tau_{c}\left\|\mathcal{P} \nabla \cdot \mathbf{u}_{h}\right\|^{2}
\end{aligned}
$$

The last two terms in (39) are positive, providing dissipation of the FE energy, but the first three could be negative, providing these models with a mechanism to predict a backward energy transfer, not frequently found in classical LES models [54]. It is justified in [21] that even if the first three terms may be negative at a certain time instant, their averaged contribution in a time window greater than the largest period needs to be positive, which is the behavior expected of backscatter from a physical point of view.

For the ASGS method, i.e., $\mathcal{P}:=\mathrm{I}$, the last term in the left hand side of (37) vanishes and the previous one reads

$$
\left(\mathcal{L}_{\mathbf{a}}\left(\mathbf{u}_{h}, p_{h}\right)+\mathcal{L}_{\mathbf{a}}^{*}\left(\mathbf{u}_{h}, p_{h}\right), \tilde{\mathbf{u}}\right)_{h}=-2\left(\nu \Delta \mathbf{u}_{h}, \tilde{\mathbf{u}}\right)_{h} .
$$

In turn, the time derivatives of the FE and subscale velocities can be combined as

$$
\frac{1}{2} d_{t}\left\|\mathbf{u}_{h}\right\|^{2}+\frac{1}{2} d_{t}\|\tilde{\mathbf{u}}\|^{2}+\left(\partial_{t} \tilde{\mathbf{u}}, \mathbf{u}_{h}\right)+\left(\partial_{t} \mathbf{u}_{h}, \tilde{\mathbf{u}}\right)=\frac{1}{2} d_{t}\left\|\mathbf{u}_{h}+\tilde{\mathbf{u}}\right\|^{2}
$$

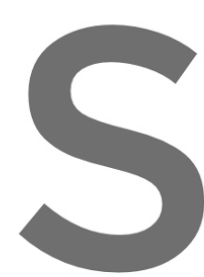

to rewrite (37) as

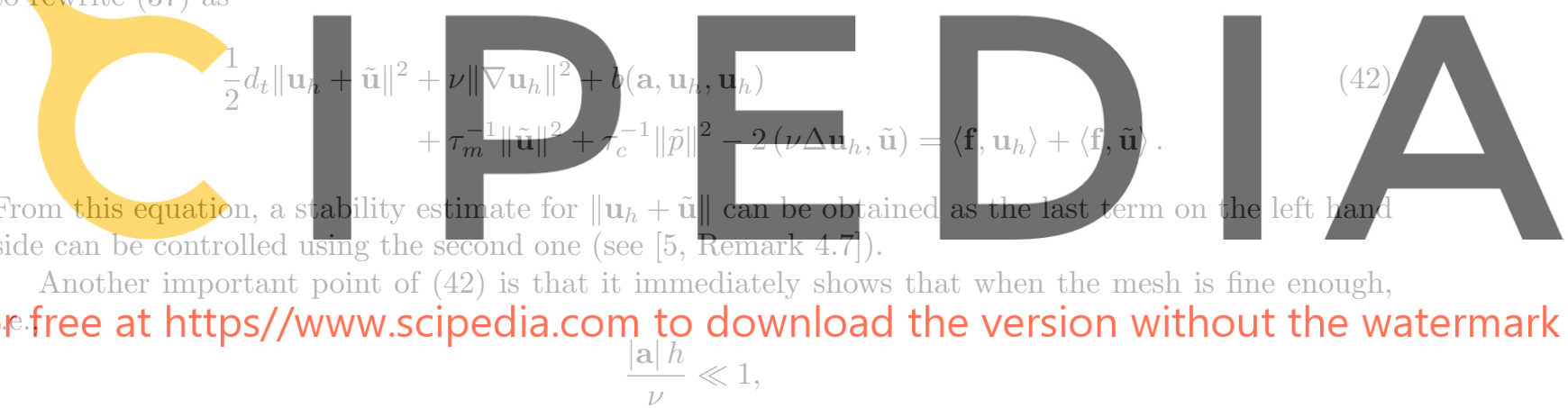

the dissipation of the total energy depends only on the viscosity. Therefore, the dissipative structure is correctly predicted when a laminar flow is considered or when the discretization is fine enough to resolve all scales of the flow, an important advantage over other LES techniques.

On the other hand, for the OSS method, the FE and subgrid kinetic energy can be summed to obtain the total one

$$
\frac{1}{2} d_{t}\left\|\mathbf{u}_{h}\right\|^{2}+\frac{1}{2} d_{t}\|\tilde{\mathbf{u}}\|^{2}=\frac{1}{2} d_{t}\left\|\mathbf{u}_{h}+\tilde{\mathbf{u}}\right\|^{2} .
$$

since $\left(\partial_{t} \tilde{\mathbf{u}}, \mathbf{u}_{h}\right)=\left(\partial_{t} \mathbf{u}_{h}, \tilde{\mathbf{u}}\right)=0$ as soon as we enforce the subscale to be orthogonal to the FE space. This property also guarantees that

$$
\begin{aligned}
\left(\Pi_{m}\left(\mathcal{L}_{\mathbf{a}}\left(\mathbf{u}_{h}, p_{h}\right)\right), \tilde{\mathbf{u}}\right) & =0 \\
\left(\Pi_{c}\left(\nabla \cdot \mathbf{u}_{h}\right), \tilde{p}\right) & =0
\end{aligned}
$$

which implies that the last term on the left hand side of (37) vanishes and that the previous one can be written as

$$
\left(\mathcal{P}\left(\mathcal{L}_{\mathbf{a}}\left(\mathbf{u}_{h}, p_{h}\right)\right)+\mathcal{L}_{\mathbf{a}}^{*}\left(\mathbf{u}_{h}, p_{h}\right), \tilde{\mathbf{u}}\right)_{h}=\left(\left(\mathcal{L}_{\mathbf{a}}\left(\mathbf{u}_{h}, p_{h}\right)+\mathcal{L}_{\mathbf{a}}^{*}\left(\mathbf{u}_{h}, p_{h}\right), \tilde{\mathbf{u}}\right)_{h}=-2\left(\nu \Delta \mathbf{u}_{h}, \tilde{\mathbf{u}}\right)_{h}\right.
$$

\footnotetext{
${ }^{2}$ However, it should be kept in mind that the numerical solution of the problem is the FE component. There is no reason to add the subscale to the final solution as the approximation is limited by the interpolation order, see [22, Remark $10]$.
} 
as in the ASGS case. Let us note that the Laplacian term can be eliminated without afecting the convergence properties of the method. Then, the global energy balance equation (37) reads

$$
\begin{aligned}
\frac{1}{2} d_{t}\left\|\mathbf{u}_{h}\right\|^{2}+\frac{1}{2} d_{t}\|\tilde{\mathbf{u}}\|^{2} & +\nu\left\|\nabla \mathbf{u}_{h}\right\|^{2}+b\left(\mathbf{a}, \mathbf{u}_{h}, \mathbf{u}_{h}\right) \\
& +\tau_{m}^{-1}\|\tilde{\mathbf{u}}\|^{2}+\tau_{c}^{-1}\|\tilde{p}\|^{2}=\left\langle\mathbf{f}, \mathbf{u}_{h}\right\rangle+(\mathcal{P}(\mathbf{f}), \tilde{\mathbf{u}}),
\end{aligned}
$$

which is exactly (42) except for the projection of the force in the last term. Stability and convergence of this formulation have been proved in $[6,7]$.

\section{Final discrete problem}

Applying a time integration algorithm to (13)-(15)-(16) we get the fully discrete problem. The final implementation of the discrete problem is written here considering a Picard linearization of the convective term and the Backward Euler (BE) scheme for the time discretization. It can be straightforwardly modified to consider the Crank-Nicolson time integration scheme; this last scheme is the one used in the numerical examples of Sections 5, 6 and 7 .

\subsection{Algebraic Subgrid Scales (ASGS)}

Taking the nonlinear advection velocity definition (25) and considering the time derivative in the fine scales, we have the Dynamic and Nonlinear ASGS method, hereinafter Dyn-Nl-ASGS. At time step

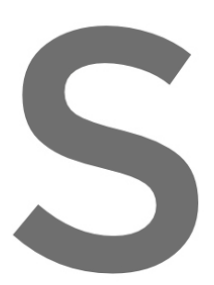
$n$ and nonlinear iteration

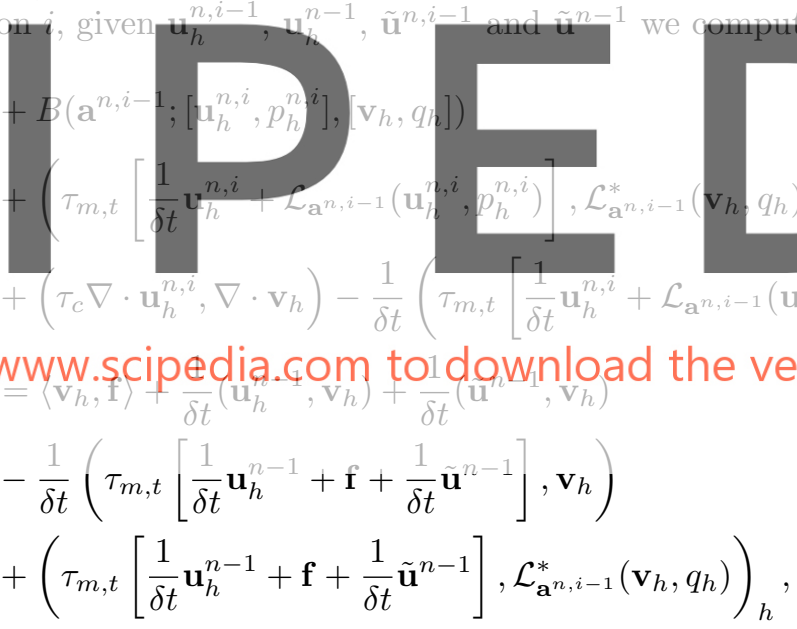

where $\tau_{m, t}=\left(\delta t^{-1}+\tau_{m}^{-1}\right)^{-1}$ and $\mathbf{a}^{n, i-1}=\mathbf{u}_{h}^{n, i-1}+\tilde{\mathbf{u}}^{n, i-1}$.

In turn, $\tilde{\mathbf{u}}^{n, i}$ is computed by solving the discretization of the fine scale problem (15). Note that in the nonlinear version of the algorithm, the stabilization parameter $\tau_{m, t}$ depends on the subscale itself through a in (20), making the fine scale equation also nonlinear, although it is local and does not increase the size of the global linear system to be solved. At each integration point of each element we iteratively solve

$$
\tilde{\mathbf{u}}^{n, i, k}=\tau_{m, t}^{k-1} \frac{1}{\delta t} \tilde{\mathbf{u}}^{n-1}+\tau_{m, t}^{k-1}\left[\mathbf{f}-\frac{\left(\mathbf{u}_{h}^{n, i}-\mathbf{u}_{h}^{n-1}\right)}{\delta t}-\mathcal{L}_{\mathbf{a}^{n, i, k-1}}\left(\mathbf{u}_{h}^{n, i}, p_{h}^{n, i}\right)\right] .
$$

where $\mathbf{a}^{n, i, k-1}=\mathbf{u}_{h}^{n, i}+\tilde{\mathbf{u}}^{n, i, k-1}$ is used in (20) to obtain $\tau_{m, t}^{k-1}$.

Alternatively, one can send the corresponding fine scale convective term $\tilde{\mathbf{u}} \cdot \nabla \mathbf{u}_{h}$ to the left-hand side, improving the convergence of the iterative process as

$$
\tilde{\mathbf{u}}^{n, i, k}+\tilde{\mathbf{u}}^{n, i, k} \cdot \nabla \mathbf{u}_{h}^{n, i}=\tau_{m, t}^{k-1} \frac{1}{\delta t} \tilde{\mathbf{u}}^{n-1}+\tau_{m, t}^{k-1}\left[\mathbf{f}-\frac{\left(\mathbf{u}_{h}^{n, i}-\mathbf{u}_{h}^{n-1}\right)}{\delta t}-\mathcal{L}_{\mathbf{u}_{h}^{n, i}}\left(\mathbf{u}_{h}^{n, i}, p_{h}^{n, i}\right)\right] .
$$

This is a simple fixed-point iterative scheme that we have found efficient and robust for the numerical simulations presented in this paper, although in other situations we have found more convenient to use a conventional Newton-Raphson scheme to solve the nonlinear subscale equation [2]. 
For the simplest ASGS scheme we do not consider the time derivative of the fine scale, we consider them quasi-static, i.e., $\left(\partial_{t} \tilde{\mathbf{u}}, \mathbf{v}_{h}\right)=0$. Note that in any case the subscales will depend on time through the finite element residual and the stabilization parameter. On the other hand, the advection velocity is considered to be linear as indicated in (24). We label this method as Static Linear ASGS (Sta-LinASGS). Note that the Sta-Lin-ASGS method does not need to explicitly compute $\tilde{\mathbf{u}}$; invoking (20) and (21) in (13) we get a discrete equation only in terms of the FE component.

We can readily define the rest of possible combinations of time and nonlinear treatment considering the linear advection velocity definition and the time-dependence in the subscales (Dyn-Lin-ASGS method) or keeping the static definition of the subscales with the nonlinear choice for the advection velocity (Sta-Nl-ASGS method).

\subsection{Orthogonal Subscales (OSS)}

Let us state the Dynamic and Nonlinear OSS (Dyn-Nl-OSS) method, which means to take into account the nonlinearity of the advection velocity (25) and the time derivative of the subscales. At time step $n$ and nonlinear iteration $i$, given $\mathbf{u}_{h}^{n, i-1}, \mathbf{u}_{h}^{n-1}, \tilde{\mathbf{u}}^{n, i-1}$ and $\tilde{\mathbf{u}}^{n-1}$ we compute $\mathbf{u}_{h}^{n, i}$ and $p_{h}^{n, i}$ solving

$$
\begin{aligned}
\frac{1}{\delta t}\left(\mathbf{u}_{h}^{n, i}, \mathbf{v}_{h}\right)+ & B\left(\mathbf{a}^{n, i-1} ;\left[\mathbf{u}_{h}^{n, i}, p_{h}^{n, i}\right],\left[\mathbf{v}_{h}, q_{h}\right]\right) \\
& +\left(\tau_{m, t}\left[\frac{1}{\delta t} \mathbf{u}_{h}^{n, i}+\mathcal{L}_{\mathbf{a}^{n, i-1}}\left(\mathbf{u}_{h}^{n, i}, p_{h}^{n, i}\right)\right], \mathcal{L}_{\mathbf{a}^{n, i-1}}^{*}\left(\mathbf{v}_{h}, q_{h}\right)\right) \\
& +\left(\tau_{c} \nabla \cdot \mathbf{u}_{h}^{n, i}, \nabla \cdot \mathbf{v}_{h}\right) \\
& =\left\langle\mathbf{v}_{h}, \mathbf{f}\right\rangle+\frac{1}{\delta t}\left(\mathbf{u}_{h}^{n-1}, \mathbf{v}_{h}\right) \\
& +\left(\tau_{m, t}\left[\mathbf{f}+\frac{1}{\delta t} \tilde{\mathbf{u}}^{n-1}-\boldsymbol{\xi}_{m}^{n, i-1}\right], \mathcal{L}_{\mathbf{a}^{n, i-1}}^{*}\left(\mathbf{v}_{h}, q_{h}\right)\right) \\
& -\left(\tau_{c} \xi_{c}^{n, i-1}, \nabla \cdot \mathbf{v}_{h}\right),
\end{aligned}
$$

where $\boldsymbol{\xi}_{m}$ and $\xi_{c}$ are the weighted projections of the residuals $\mathbf{R}_{u}$ and $R_{p}$ (see below) evaluated at the corresponding time step and nonlinear iteration.

Like the Dyn-NL-ASGS method, we also need to compute the subscale velocity $\tilde{\mathbf{u}}$ explicitly. We compute the discrete subscale problem with the OSS counterpart of (49) or (50), viz.

$$
\begin{aligned}
\tilde{\mathbf{u}}^{n, i, k} & +\tilde{\mathbf{u}}^{n, i, k} \cdot \nabla \mathbf{u}_{h}^{n, i}=\tau_{m, t}^{k-1} \frac{1}{\delta t} \tilde{\mathbf{u}}^{n-1} \\
& +\tau_{m, t}^{k-1}\left[\mathbf{f}-\frac{\left(\mathbf{u}_{h}^{n, i}-\mathbf{u}_{h}^{n-1}\right)}{\delta t}-\mathcal{L}_{\mathbf{u}_{h}^{n, i}}\left(\mathbf{u}_{h}^{n, i}, p_{h}^{n, i}\right)\right]-\tau_{m, t}^{k-1} \boldsymbol{\xi}_{m}^{n, i-1}
\end{aligned}
$$

Note that $\boldsymbol{\xi}_{m}^{n, i}$ actually depends on $\tilde{\mathbf{u}}^{n, i}$ via the advection velocity of the convective term $\mathbf{a}^{n, i}=\mathbf{u}_{h}^{n, i}+$ $\tilde{\mathbf{u}}^{n, i}$. In order to simplify the fine scale computation (52) we use the projection at the previous nonlinear iteration, i.e., $\boldsymbol{\xi}_{m}^{n, i-1}$.

For the dynamic OSS case we should introduce some modifications in the computation of the projection $\Pi_{m}$. At the fully discrete level, in order for $\tilde{\mathbf{u}}^{n+1}$ to be $L^{2}$ orthogonal to $\mathcal{V}_{h}$, we must add to the FE residual the subscale time derivative contribution from the previous time step and use $\tau_{m, t}$ instead of $\tau_{m}$ in the computation of the projections. Finally, the projections of the residuals onto the FE spaces $\boldsymbol{\xi}_{m}^{n, i}$ and $\xi_{c}^{n, i}$ are such that

$$
\begin{array}{ll}
\left(\tau_{m, t} \boldsymbol{\xi}_{m}^{n, i}, \mathbf{v}_{h}\right)=\left(\tau_{m, t}\left(\mathbf{R}_{u}^{n, i}+\frac{1}{\delta t} \tilde{\mathbf{u}}^{n-1}\right), \mathbf{v}_{h}\right) & \forall \mathbf{v}_{h} \in \mathcal{V}_{0, h}, \\
\left(\tau_{c} \xi_{c}^{n, i}, q_{h}\right)=\left(\tau_{c} R_{p}^{n, i}, q_{h}\right) & \forall q_{h} \in \mathcal{Q}_{0, h},
\end{array}
$$

where the residuals $\mathbf{R}_{u}^{n, i}$ and $R_{p}^{n, i}$ are evaluated using (17) and (18) with $\mathbf{a}^{n, i}=\mathbf{u}_{h}^{n, i}+\tilde{\mathbf{u}}^{n, i}, \mathbf{u}_{h}^{n, i}, \mathbf{u}_{h}^{n-1}$ and $p_{h}^{n, i}$. Note that when convergence of the nonlinear iteration is achieved, (52) and (53) guarantee that $\left(\mathbf{v}_{h}, \tilde{\mathbf{u}}^{n}\right)=0$ for any $\mathbf{v}_{h} \in \mathcal{V}_{0, h}$. 


\section{Decay of homogeneous isotropic turbulence}

\subsection{Problem definition}

This problem, one of the most used benchmarks to test LES models, consists of analysing the statistics of the turbulent flow in a 3D box of size $\Omega=(0,2 \pi) \times(0,2 \pi) \times(0,2 \pi)$ with periodic boundary conditions in all directions, which is started with a field having a predetermined energy spectra. We solve this problem using the different VMS models of Section 4 and structured meshes with $N_{x} \times N_{y} \times N_{z}$ linear, quadratic and cubic hexahedral elements, taking $\left(N_{x}, N_{y}, N_{z}\right)=(32,32,32),(64,64,64)$ and $(128,128,128)$.

\subsubsection{Initial condition}

The initial energy spectra [46] is given by

$$
E(k, 0)=\frac{q^{2}}{2 A} k_{0}^{-(\sigma+1)} k^{4} \exp \left(-\frac{\sigma}{2}\left(\frac{k}{k_{0}}\right)^{2}\right)
$$

where $k_{0}$ is the wave number at which $E(k, 0)$ is maximum, $q^{2} / 2=3 / 2$ is the total kinetic energy, $\sigma=4$ and $A=\int_{0}^{\infty} k^{\sigma} \exp \left(-\sigma k^{2} / 2\right)=\frac{3}{32} \sqrt{\frac{\pi}{2}}$. Following Rogallo [53], we generate the initial field on the Fourier space such that it satisfies continuity and has the energy spectrum prescribed in (55). We finally transform the initial field to the physical space applying the inverse Fast Fourier Transform. We compare the results agains those available in [1], so we set $k_{0}=6$ and the viscosity such that the associated Taylor-microscale Reynolds number is $\operatorname{Re}_{\lambda}=952$, which results in $\nu=3.5014006 \cdot 10^{-4}$. The initial analytical energy spectrum and the one computed in a $128^{3} Q 1$ elements mesh are shown in Fig. 1

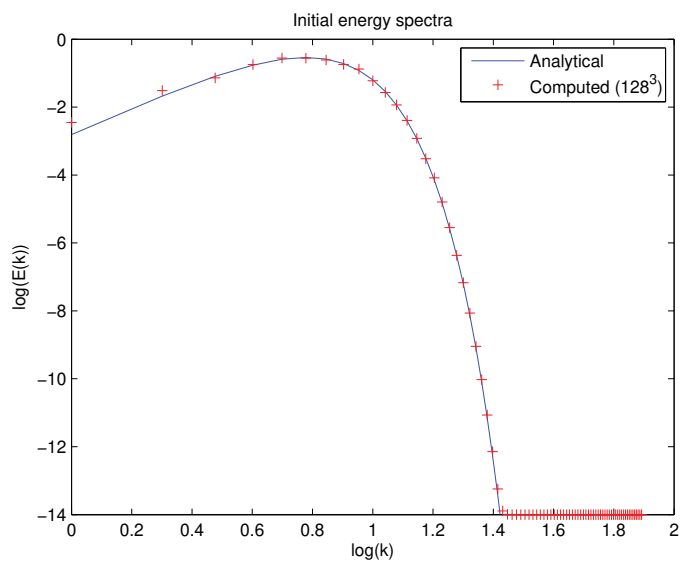

Figure 1: Analytical and computed initial energy spectra

\subsubsection{Setting}

We test the ASGS method and the OSS method reported in Section 4. The problem is solved considering three different cases for both methods, depending on the definition of the advection velocity and the tracking of the subscales. The advection velocity a can be linear or nonlinear and the subscales can be dynamic or static (see Subsection 2.2). Table 1 collects all the VMS combinations to be compared for the different simulations.

In terms of the numerical parameters of the methods, we use the skew-symmetric convective term type 1 defined in Subsection 2.1. The stabilization parameter $\tau_{c}$ is set equal to zero and the algorithmic constants that concerns on $\tau_{m}$ are $c_{1}=12$ and $c_{2}=2$, see Section 8 . Further, we use linear, quadratic and cubic FEs.

The time integration has been performed using the Crank-Nicolson scheme with an adaptive time step. The initial time step is set to $\delta t_{0}=5.0 \cdot 10^{-3}$ and it is increased at each step multiplying it by 


\begin{tabular}{ccccc}
\hline Case Id. & Label & Method & Advection velocity $(\mathbf{a})$ & Subscales tracking \\
\hline 1 & Sta-Lin-ASGS & ASGS & Linear $\left(\mathbf{a}=\mathbf{u}_{h}\right)$ & Static $\left(\partial_{t} \tilde{\mathbf{u}}=\mathbf{0}\right)$ \\
2 & Dyn-Lin-ASGS & ASGS & Linear $\left(\mathbf{a}=\mathbf{u}_{h}\right)$ & Dynamic $\left(\partial_{t} \tilde{\mathbf{u}} \neq \mathbf{0}\right)$ \\
3 & Dyn-Nl-ASGS & ASGS & Nonlinear $\left(\mathbf{a}=\mathbf{u}_{h}+\tilde{\mathbf{u}}\right)$ & Dynamic $\left(\partial_{t} \tilde{\mathbf{u}} \neq \mathbf{0}\right)$ \\
\hline 4 & Sta-Lin-OSS & OSS & Linear $\left(\mathbf{a}=\mathbf{u}_{h}\right)$ & Static $\left(\partial_{t} \tilde{\mathbf{u}}=\mathbf{0}\right)$ \\
5 & Dyn-Lin-OSS & OSS & Linear $\left(\mathbf{a}=\mathbf{u}_{h}\right)$ & Dynamic $\left(\partial_{t} \tilde{\mathbf{u}} \neq \mathbf{0}\right)$ \\
6 & Dyn-Nl-OSS & OSS & Nonlinear $\left(\mathbf{a}=\mathbf{u}_{h}+\tilde{\mathbf{u}}\right)$ & Dynamic $\left(\partial_{t} \tilde{\mathbf{u}} \neq \mathbf{0}\right)$ \\
\hline
\end{tabular}

Table 1: DHIT test cases

an amplification factor. For this test the amplification is equal to 1.1 , reading $\delta t_{i}=1.1 \cdot \delta t_{i-1}$. The time step is increased step by step until it reaches a predefined threshold, e.g., $0.1 \mathrm{~s}$. If convergence is not attained at either the nonlinear or solver loop we apply a reduction factor (5.0 for this test) and recalculate the solution using the reduced time step, i.e., $\delta t_{i}=\frac{1}{5.0} \delta t_{i-1}$. The amplification of the time step when the solution converges allows one to decrease the total amount of time steps needed for the computation. At each time step the nonlinear system is solved as described in Section 4.

\subsection{Numerical results}

\subsubsection{Energy Conservation}

In this section we present results of the energy budget described in Section 3 obtained in a $32^{3}$ elements mesh for the ASGS and OSS methods using the dynamic and nonlinear cases. Fig. 2 depicts the energy balance evolution for the mean flow equation (35) and the subscale equation (36) separately for the Dyn-Nl-ASGS case. It can be seen that the variation of kinetic energy shown by the FE component in Fig. 2(a) is offset in a large part by the transfer of energy to the subscales, while remaining energy on the mean flow balance is offset by the viscous term. On the other side, Fig. 2(b) shows that the energy transferred from the FE equation is mainly dissipated by the subscale velocity term. There is an small variation of the kinetic energy of the subscale at the beginning of the simulation. Note that since the viscosity is small, so are the viscous effects compared to the dissipation introduced by the subscale velocity. As we use a skew-symmetric form of the convective term, this term does not affect the energy balance and is not plotted in Fig. 2(a). Since $\tau_{c}=0$, the pressure subscale term $\tau_{c}^{-1}\|\tilde{p}\|^{2}=\tau_{c}\left\|\mathcal{P}\left(\nabla \cdot \mathbf{u}_{h}\right)\right\|^{2}$ is also zero and does not appear in Fig. 2(b).

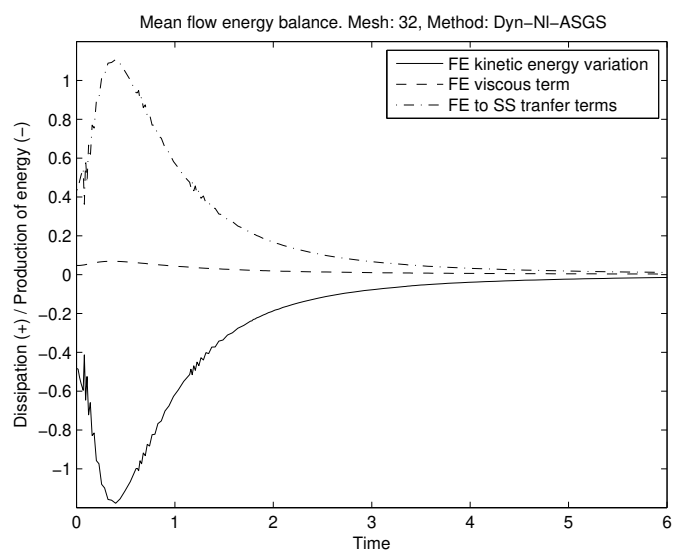

(a) Mean flow equation energy balance

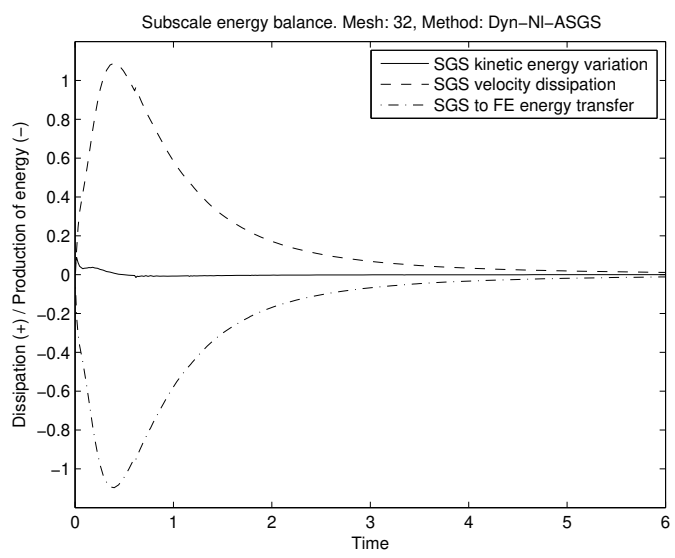

(b) Subscale equation energy balance

Figure 2: Mean flow and subscale energy balances

The energy balance evolution for the mean flow and the subscales equations in (35)-(36) for the Dyn-Nl-OSS case are shown in Fig. 3. Fig. 3(a) depicts the energy balance evolution for the mean flow equation. Like the ASGS method, the loss of kinetic energy is balanced by the FE to subscales energy transfer terms. The FE viscous term also has a very little impact on the dissipation of energy. On the 
other side, the subscales energy balance shown in Fig. 3(b) shows that almost all the energy transferred by the FE to the subscales is offset by the subscale velocity term, again like in the ASGS method. The only important difference between both methods is that no oscillations are observed in the FE kinetic energy evolution when the OSS method is used.

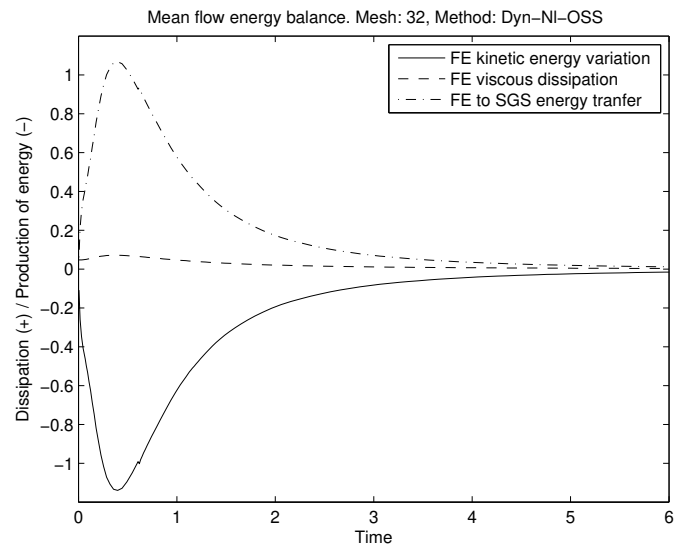

(a) Mean flow equation energy balance

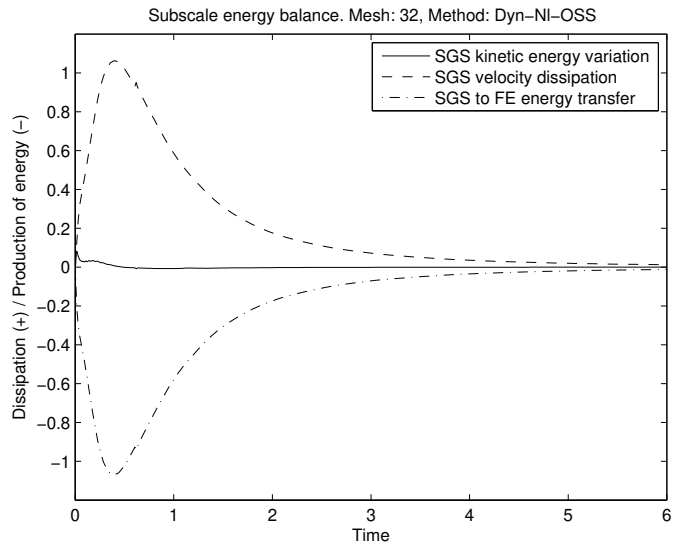

(b) Subscale equation energy balance

Figure 3: Mean flow and subscale energy balances

The global energy balance terms obtained solving the problem with the skew-symmetric convective term type 2 for the Dyn-Nl-ASGS and Dyn-Nl-OSS cases is shown in Fig. 4. We note that the loss of skew-symmetry in the convective term has a non-negligible effect (see Figs.4(a) and 4(b)). In particular, this term introduces negative dissipation (production of energy) into the problem. As we will show in Section 5.2.3, this fact implies that the method is less dissipative and the energy decays in a slower rate than using the convective term type 1 . This negative contribution, however, is not predictable and could result in a blow up of the calculation. We refer to Section 8 for some comments about numerical instabilities associated to the type 2 convective term.

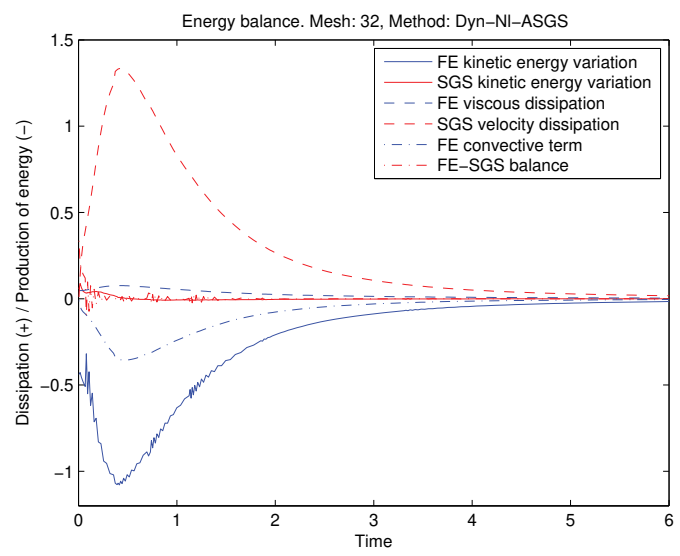

(a) Global energy balance for Dyn-Nl-ASGS

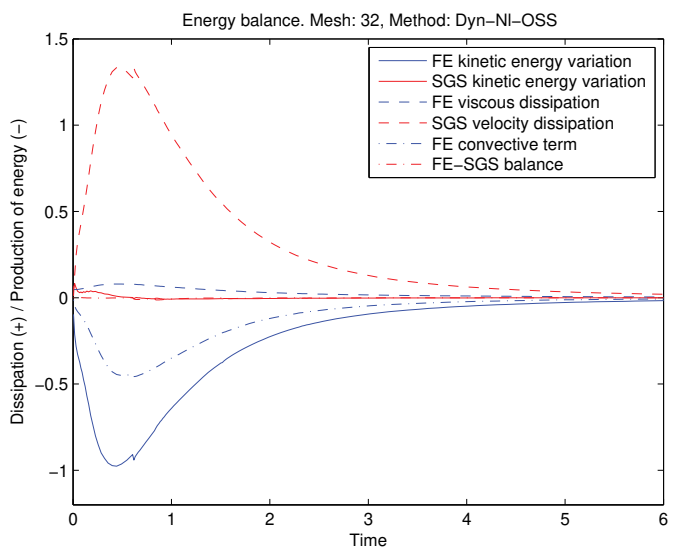

(b) Global energy balance for Dyn-Nl-OSS

Figure 4: Global energy balance using skew-symmetric convective term type 2

\subsubsection{Computational costs analysis}

The actual implementation in the parallel FE multiphysics code FEMPAR [8] is based on a classical domain decomposition strategy. At each nonlinear iteration the monolithic linear system is solved using 
a classical GMRES method applied to the Schur complement over the interfaces of the subdomains. This iterative procedure is preconditioned using a balancing Neumann-Neumann method applied to the monolithic system. The cost of each iteration is that of local Dirichlet solves for the Schur complement application and a local Neumann solve and a global solve for the preconditioner application (see [45, 23, 9]). All local systems are solved using the sparse direct solvers in PARDISO library [55, 56].

An important issue when comparing different computational methods is their corresponding computational cost. In order to characterize the performance of the different VMS methods introduced in Section 2, we analyze some quantities that define the computational cost of each method, viz. nonlinear iterations, iterative solver iterations and the adaptive time step evolution.

All cases compared here have been solved using a $32^{3}$ linear hexahedral element mesh. This discretization is very coarse and it allows us to stress the differences between the proposed methods. In fact, due to this discretization, the linear and static ASGS case (Sta-Lin-ASGS) and the dynamic and linear ASGS case (Dyn-Lin-ASGS) do not converge at $t=0.0$ and $t=0.123$, respectively; the nonlinear iterations diverge even reducing the time step size. Anyway, all the methods converge as $h \rightarrow 0$.

The number of nonlinear iterations needed at each time step by the ASGS method is smaller than the one required by the OSS method in all cases. This is due to the evaluation of the projections at the previous nonlinear iteration $i-1$; the implicit treatment of the projection is carried out by the nonlinear loop. Alternatively, since the projection is a linear operation, it can be performed together with the linear system [20], although a more involved implementation is required. Referring to the OSS method, we observe that the dynamic cases, both linear and nonlinear, need less iterations to achieve convergence without any significant difference between each other.

However, the number of nonlinear iterations is not the only measure of the computational cost as the cost of each iteration is not fixed when iterative linear solvers are considered. Fig. 5(a) shows the maximum number of solver iterations for each time step for the methods that have attained convergence. Unlike the nonlinear iterations, here we see that the ASGS method requires more solver iterations than OSS. The maximum solver iterations at each time step for the dynamic and nonlinear ASGS case is variable, starting from near 600, dropping to 200 and rising to around 300 iterations at the end of the computation. Meanwhile, all cases of the OSS method seem to remain almost constant, around 60 iterations in the dynamic cases and around 40 iterations in the static one.

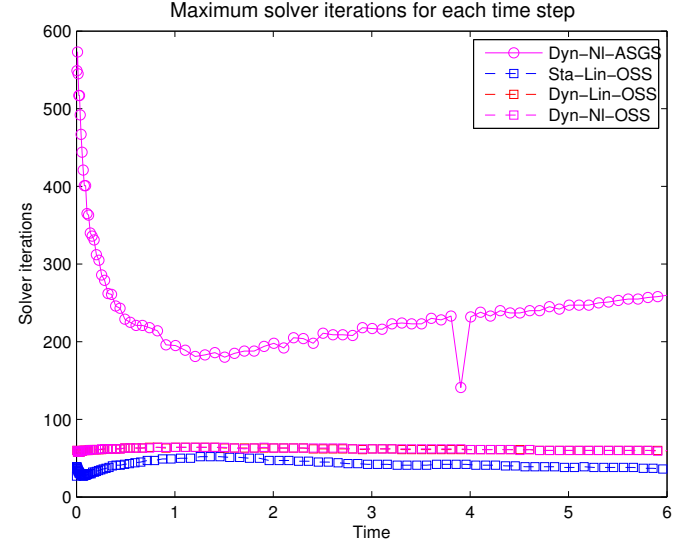

(a) Maximum solver iterations at each time step

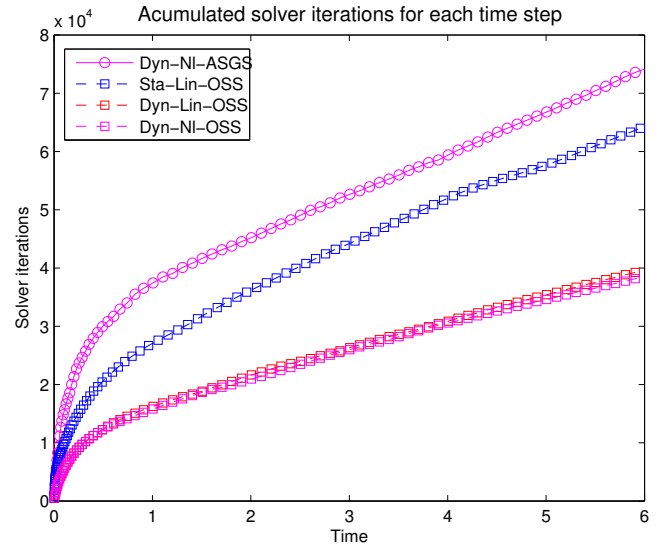

(b) Accumulated solver iterations at each time step

Figure 5: Maximum and accumulated solver iterations

The adaptive time stepping described in the previous subsection has an important role on the computational cost, as mentioned earlier. If the time step is reduced in order to ensure convergence, the global computational cost is increased. Then, we are looking for those methods that do not require time step reductions, consequently reducing the total amount of time step evaluations. In this case, any of the methods shown in Fig. 5 need to reduce the time step. Since we do not have any time step reduction and the number of solver iterations per step is stabilized after $t=1$, the total amount of accumulated 
solver iterations (in nonlinear and time loops) shown in Fig. 5(b) increases almost linearly. We see in this figure that the ASGS scheme performs worse than OSS counterparts in this aspect. With respect to the OSS method, we see that the number of nonlinear iterations needed by the static version of this method results in a steeper slope of the accumulated solver iterations. No significant differences appear between the dynamic linear and nonlinear definitions of the OSS method.

Summarizing, ASGS methods need less nonlinear iterations, but on the other hand OSS methods need less solver iterations. On the other hand, ASGS formulations are prone to instabilities; linear formulations diverge and the nonlinear dynamic formulation requires much more solver iterations.

We can clearly state that the most efficient method for this setting, in terms of computational cost, is the dynamic (both linear and nonlinear) OSS method; all OSS cases are below ASGS. It has to be said that the dynamic nonlinear OSS case requires less nonlinear iterations in some of the time step computations.

\subsubsection{Total energy evolution}

In this section we present the total energy evolution of the resolved scales, i.e., the FE component. The results are shown in Fig. 6(a). We observe that all methods have a very similar performance for this test case, still far from the DNS result. Note that we do not plot the non-converged results from the ASGS static cases.

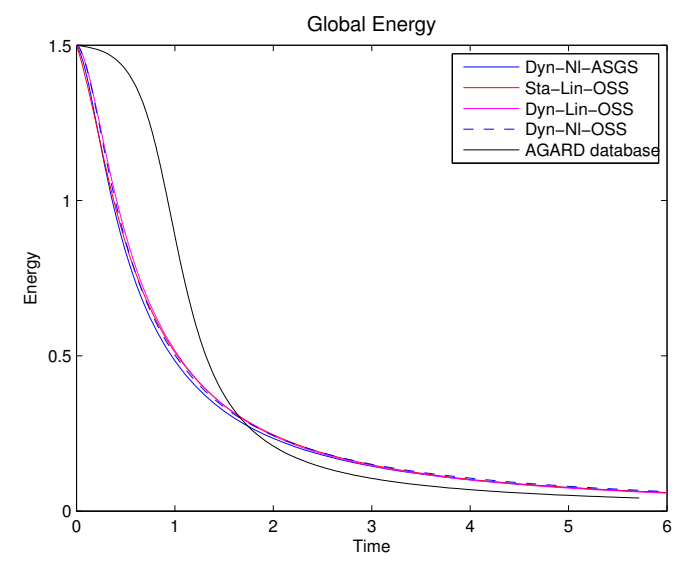

(a) Using the skew-symmetric term type 1

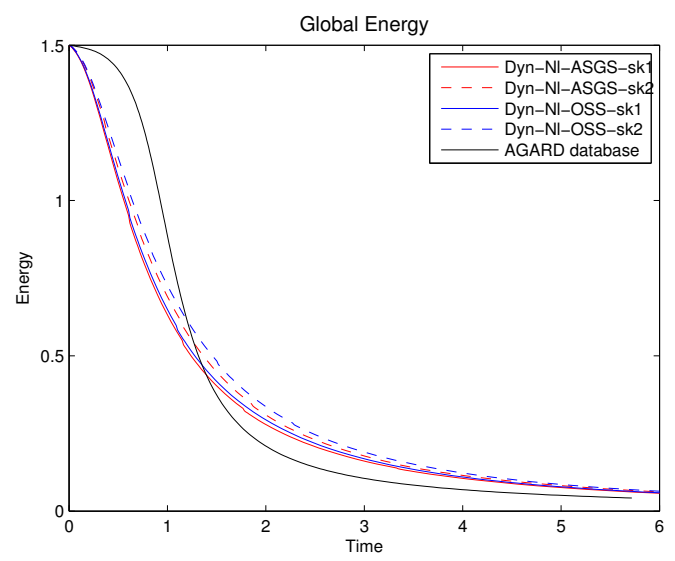

(b) Using the skew-symmetric term type 2

Figure 6: Total energy evolution for a $32^{3}$ elements mesh with the scaled initial condition

The results shown so far have been obtained using the skew-symmetric convective term type 1 defined in (10). We test the influence of this term by solving the same problem with the convective skew-symmetric term (type 2) in (11) for dynamic and nonlinear ASGS and OSS schemes. The results obtained for this test are shown in Fig. 6(b), where it is seen that the solution using the type 2 term is above the one obtained with term type 1, which shows that the convective term is actually productive.

\subsubsection{Energy spectra}

According to [46], for a DNS in an isotropic decaying turbulence problem, the resolution of the small scales is judged by the shape of the energy spectra at high wave numbers and requires $k_{\max } \eta \approx 1$, $\eta=\left(\nu^{3} / \epsilon\right)^{1 / 4}$ being the Kolmogorov length scale. In this case $k_{\text {max }} \approx 182$, which means at least a $300^{3}$ FE mesh for a DNS computation, with a very important computational cost. In this section we evaluate the capability of the VMS method to represent the energy of the eddies at the inertial subrange without solving the small scales and compare the results against Kolmogorov's law prediction

$$
E(k) \propto \epsilon^{2 / 3} k^{-5 / 3},
$$

$\epsilon$ being the turbulent energy dissipation and $k$ the turbulent kinetic energy. 
In Fig. 7 the energy spectra at time $t=0.2$ for the different cases described in Table 1, excluding the non-converged ones, using a $32^{3}$ linear hexahedral element mesh is presented. We can see in Fig. 7 that the energy spectra at $t=0.2$ decays with a different slope depending on the VMS method used. Although the differences are small and only appear at large wavenumbers, we see that the dynamic OSS models are less dissipative than the Dyn-Nl-ASGS and Sta-Lin-OSS ones.

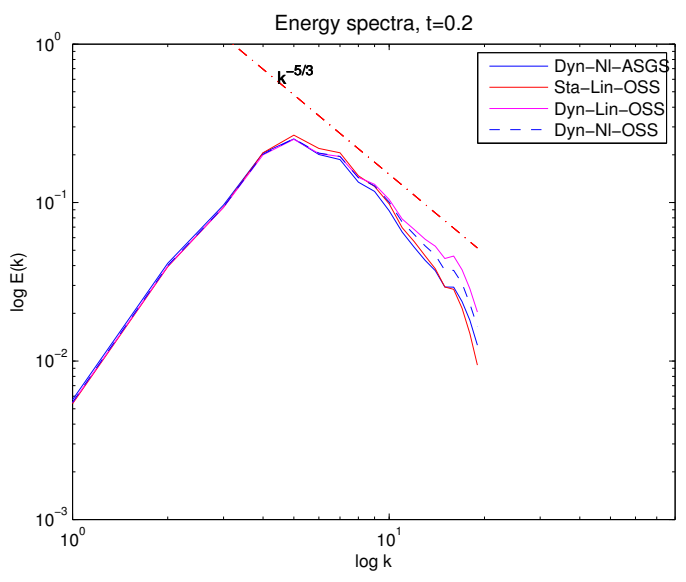

Figure 7: Energy spectra at $t=0.2$

\subsection{5. h-p refinement}

The energy decay computed using a $32^{3}$ linear FE mesh is far from the one obtained using DNS [1], as shown in Fig. (6(a)) and discussed above. To make clear that these poor results are due to this crude discretization, we present a mesh refinement analysis, both reducing the element length $h$ and increasing the interpolation order $p$. We choose the Dynamic and Nonlinear OSS method (Dyn-Nl-OSS), which is the one that shows the lowest slope in the accumulated iterations evolution (Fig. 5(b)) for the $32^{3}$ linear elements mesh. We solve the problem using the discretizations exposed in Table 2 .

\begin{tabular}{ccc}
\hline Label & Mesh elements & Element type \\
\hline $32(Q 1)$ & $32^{3}$ & hexahedral linear $(Q 1)$ \\
$64(Q 1)$ & $64^{3}$ & hexahedral linear $(Q 1)$ \\
$128(Q 1)$ & $128^{3}$ & hexahedral linear $(Q 1)$ \\
$32(Q 2)$ & $32^{3}$ & hexahedral quadratic $(Q 2)$ \\
$64(Q 2)$ & $64^{3}$ & hexahedral quadratic $(Q 2)$ \\
$32(Q 3)$ & $32^{3}$ & hexahedral cubic $(Q 3)$ \\
\hline
\end{tabular}

Table 2: $h-p$ refinement cases

In Fig. (8) we show the total kinetic energy evolution obtained using the discretizations defined in Table 2. Reducing the mesh size $h$ and/or increasing the polynomial order $p$ (not to be confused with the pressure) the result becomes closer to the DNS, as expected. In Fig. 8(b) three groups can be clearly observed, namely $32(Q 1), 32(Q 2)$ and $64(Q 1)$ and the remaining three. The best results are obtained using $Q 2$ elements although the difference is really small.

Given the differences in the total energy evolution the time at which the $k^{-5 / 3}$ law is achieved differs for the different methods. We show the energy spectra at time $t=0.2, t=0.4$ and $t=0.8$ for the different cases presented before in Fig. 9. As it can be observed in Fig. 9(a), at $t=0.2$, the energy spectra obtained using the $32(Q 1)$ mesh is almost parallel to the $k^{-3 / 5}$ line, while for the other cases low wave numbers are still being activated, as expected (kinetic energy decay occurs earlier in the 32 (Q1) case). At $t=0.4$, as shown in Fig. 9(b), the 32 (Q1) case has the right energy decay slope, while for the cases with more degrees of freedom the contribution of the small scales to the energy is still increasing. Finally, Fig. 9(c) shows us that the energy spectra for all cases has a proper energy cascade at $t=0.8$. 


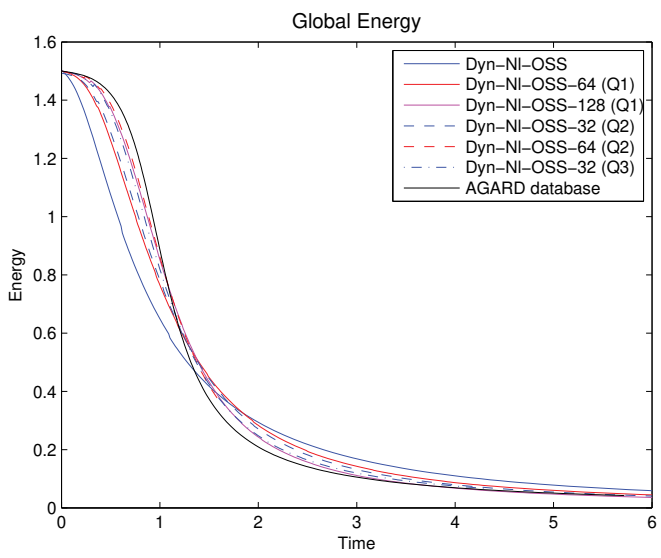

(a) Total evolution

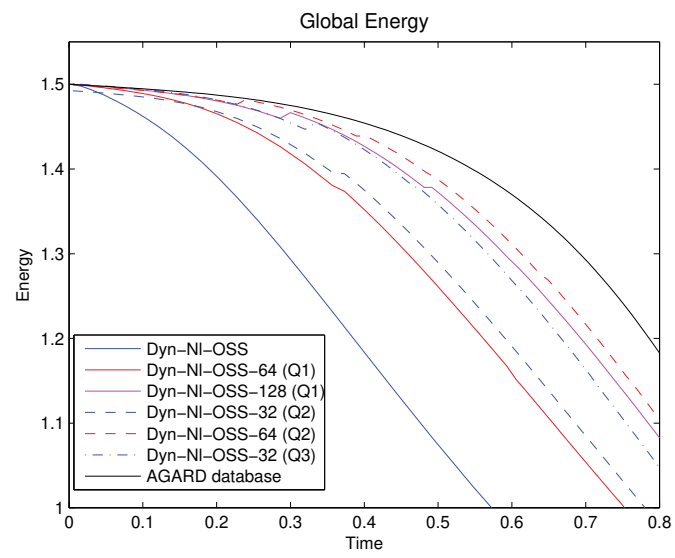

(b) Close up view

Figure 8: Total kinetic energy evolution

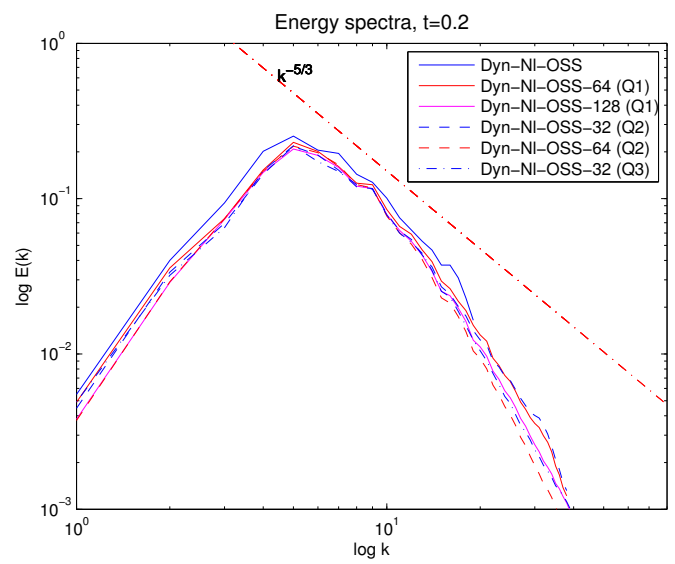

(a) Energy spectra at $t=0.2$

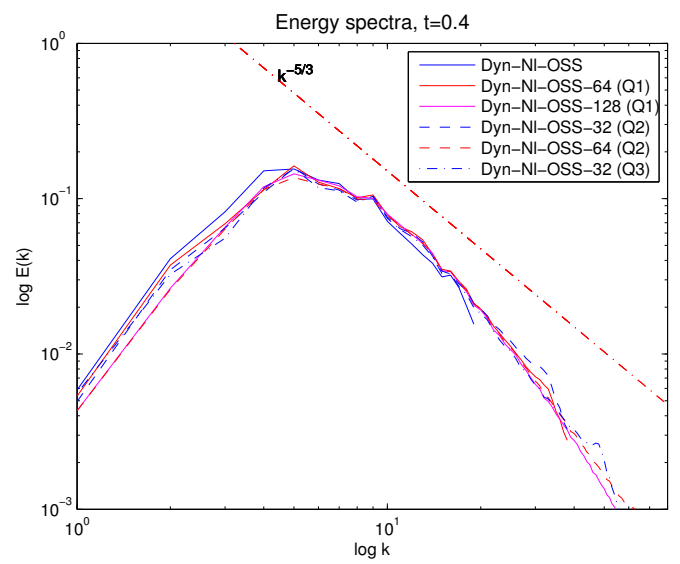

(b) Energy spectra at $t=0.4$

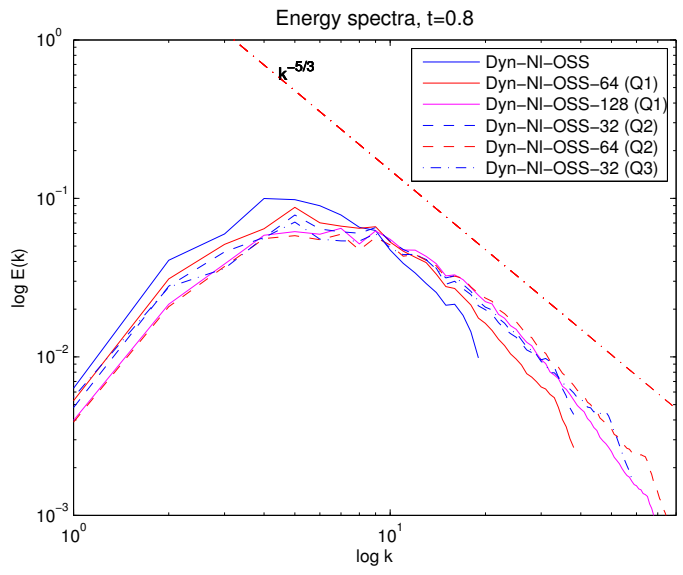

(c) Energy spectra at $t=0.8$

Figure 9: Energy spectra at different times for the $h-p$ refinement discussion 


\subsubsection{Comparison with a non-stabilized method}

All the results presented up to this point have been computed using a VMS method, either ASGS or OSS. But, what would be the result using other methods? Are the methods presented here, comparable with classical LES methods? Which methods perform better? To answer all these questions, we have solved the problem adding a physical model without stabilization. To do that, an inf-sup stable element is needed and we have used the Taylor-Hood $Q 2 / Q 1$ element. We use the classical static Smagorinsky model, consisting in adding a turbulent viscosity $\nu_{t}$ that depends on the velocity gradient and the characteristic element length $h$. This additional viscosity also acts as stabilization of convection, as usual in standard LES simulations. Then, we have to solve the standard Galerkin problem using TaylorHood $Q 2 / Q 1$ elements and introducing a modified viscosity defined as

$$
\nu=\nu_{l}+\nu_{t},
$$

where $\nu_{l}$ is the real flow viscosity and $\nu_{t}=\left(C_{s} h\right)^{2}\left|\nabla^{s} \mathbf{u}\right| . C_{s}$ is the Smagorinsky constant, which we set equal to 0.15 .

In Fig. 10 we show the total kinetic energy time evolution and the energy spectra of this method compared with the dynamic and nonlinear OSS method. In particular, we show the results for those cases from Table 2 such that they have $65^{3}$ velocity nodes. We can see in Fig. 10(a) that the total kinetic energy is decaying faster for the non-stabilized method than for the OSS method. This behavior is directly related to the shape of the energy spectra in Figs. 10(b)-10(d), where we can see that the Smagorinsky method presents lower values of energy at $t=0.4$. It is important to point out the pile-up that appears in the Smagorinsky spectra, denoting that small scales are not dissipating energy properly.

\section{Taylor-Green Vortex}

\subsection{Problem definition}

The Taylor-Green vortex (TGV) problem is a typical and widely used problem in turbulence numerical simulations. This problem aims to show, in a relatively simple flow, the basic turbulence decay

mechanisms like the turbulent energy cascade, the production of small eddies and the enhancement of dissipation by the stretching of vortex lines.

\subsubsection{Initial condition}

As in the previous example, the computational domain is the unit cube with periodical boundary conditions. The initial analytical condition for this problem, unlike the DHIT problem, is defined in the physical space (see, e.g., [28]), and given by

$$
\begin{aligned}
& u_{x}=u_{0} \cos (x) \sin (y) \sin (z), \\
& u_{y}=-u_{0} \sin (x) \cos (y) \sin (z), \\
& u_{z}=0, \\
& p=p_{0}+\frac{1}{16}(\cos (2 x)+\cos (2 y))(\cos (2 z)+2),
\end{aligned}
$$

with

$$
u_{0}=\frac{2}{\sqrt{3}} \sin \left(\gamma+\frac{2 \pi}{3}\right) .
$$

We choose $\gamma=0$, which gives the mean initial velocity $u_{0}=1$. The initial velocity field on the Fourier space has eight modes located at $\mathbf{k}=( \pm 1, \pm 1, \pm 1)$, which means that the initial flow generates a single vortex scale. The pressure constant parameter $p_{0}$ is chosen equal to zero.

\subsubsection{Setting}

We solve the TGV problem using a Reynolds number $\mathrm{Re}=1,600$. The most common Reynolds numbers available in the literature are $\operatorname{Re}=800, \operatorname{Re}=1,600$ and $\mathrm{Re}=3,000$ (see, e.g., [11, 25, 28, 41]). We use the same VMS methods as for the DHIT problem defined in Section 5 to solve this test, namely the ASGS and OSS methods, both with linear and nonlinear definitions of the convective term and 


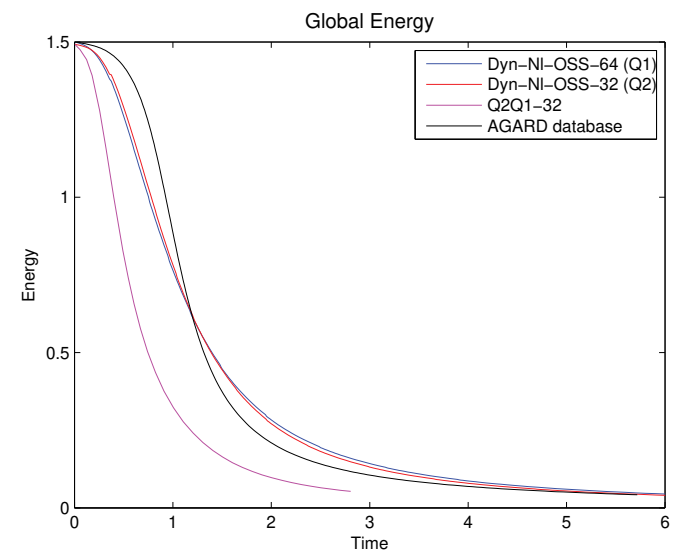

(a) Energy evolution

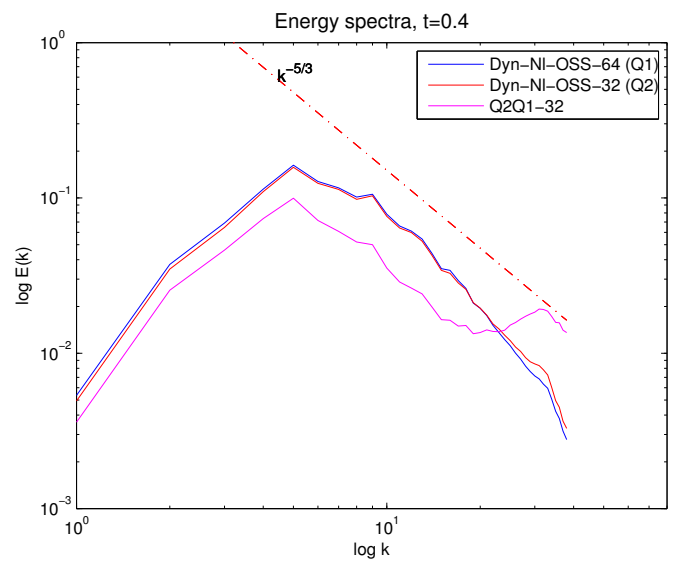

(c) Energy spectra at $t=0.4$

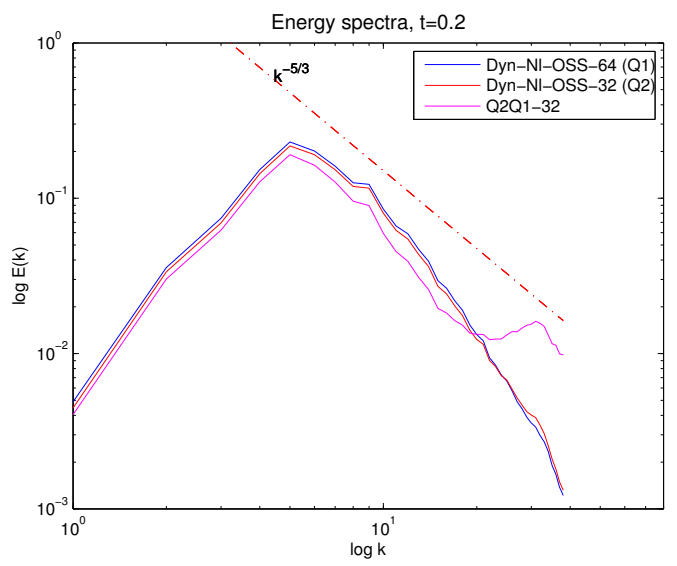

(b) Energy spectra at $t=0.2$

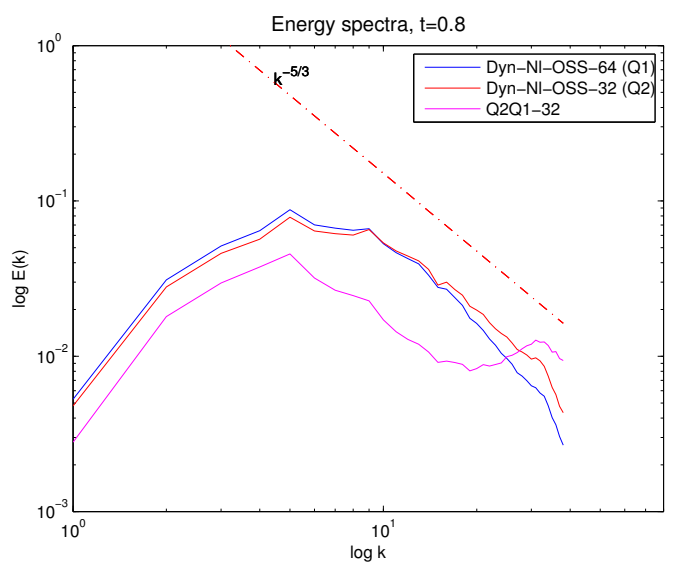

(d) Energy spectra at $t=0.8$

Figure 10: Total kinetic energy evolution and energy spectra using OSS and non-stabilized method with an inf-sup stable $Q 2 / Q 1$ element

static or dynamic tracking in time of the subscales, as it is summarized in Table 1. The stabilization parameters for each method are the same chosen for the DHIT test, see Subsection 5.1.2, and discussed in Section8.

Initially we consider a mesh of $32^{3}$ hexahedral linear elements $(Q 1)$, but we will redefine this discretization to analyze the method performance when we refine the mesh, decreasing the element size $h$ or increasing the degree of the interpolation polynomial $p$. That will imply to solve the problem on meshes with $64^{3}$ and $128^{3}$ linear $(Q 1)$, quadratic $(Q 2)$ or cubic $(Q 3)$ hexahedral elements.

\subsection{Numerical results}

\subsubsection{Vorticity}

The TGV test is characterized by its laminar evolution at the initial time steps, when the flow is strongly anisotropic due to the structured large-scale vortices directly related to the initial condition. If the Reynolds number is large enough, the vortex-stretching process, which activates the energy cascade effect, transfers energy from large to small-scales and the flow becomes unstable and turbulent. According to Brachet et al. [13], the flow becomes nearly isotropic for $\operatorname{Re} \geq 1,000$.

In Fig. 11 we present some vorticity isosurface images showing this process for a $128^{3}$ linear hexhaedral elements mesh, for the dynamic and nonlinear OSS method. Note that the initial condition (Fig. 11(a)) consists in eight vortices with the same scale corresponding to the eight Fourier modes located at $\mathbf{k}=( \pm 1, \pm 1, \pm 1)$, as it has been pointed out previously. 


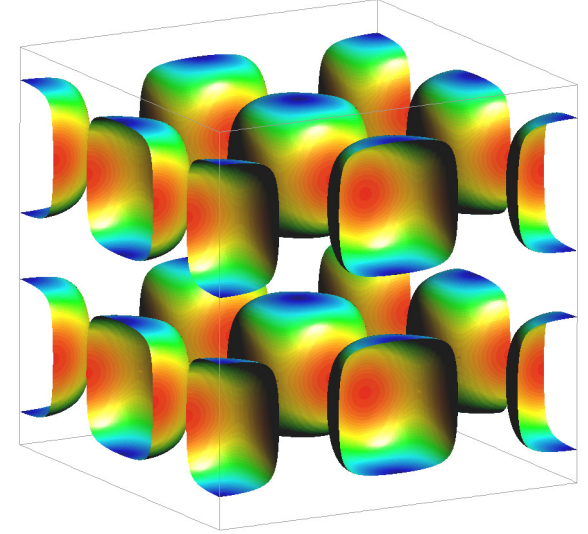

(a) Isosurface for $|\omega|=1.0$ at $t=0.0$

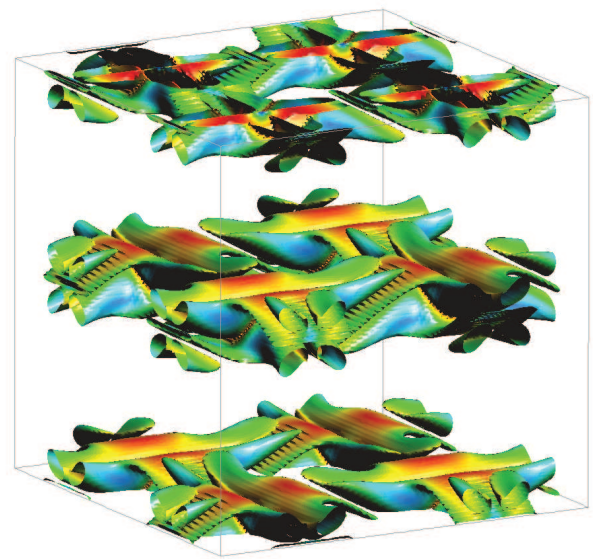

(c) Isosurface for $|\omega|=2.5$ at $t=4.1$

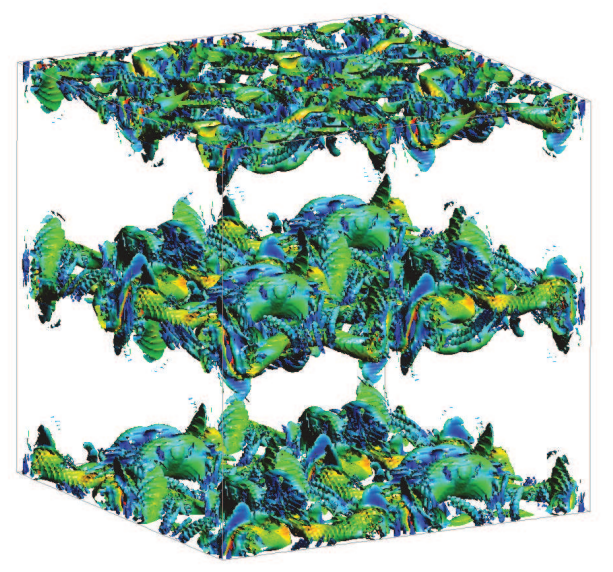

(e) Isosurface for $|\omega|=8.0$ at $t=8.2$

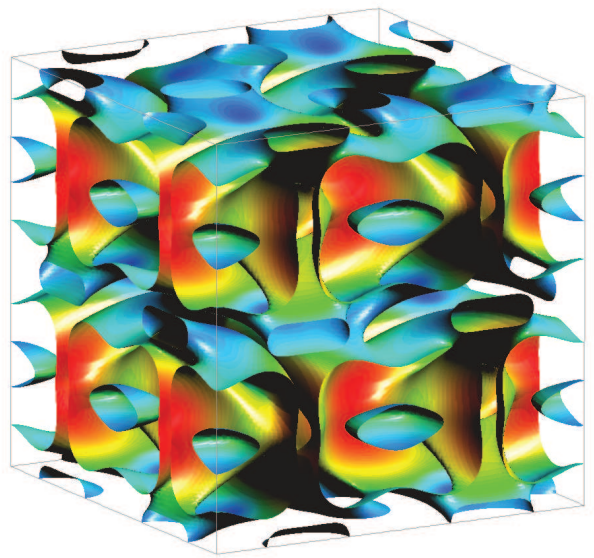

(b) Isosurface for $|\omega|=1.0$ at $t=2.0$

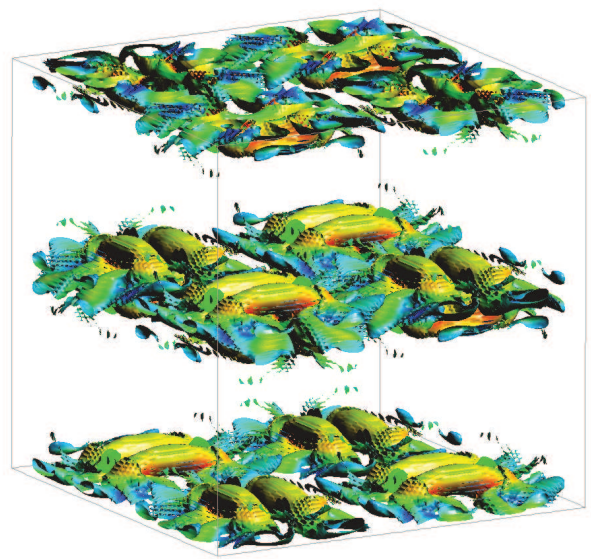

(d) Isosurface for $|\omega|=5.0$ at $t=6.1$

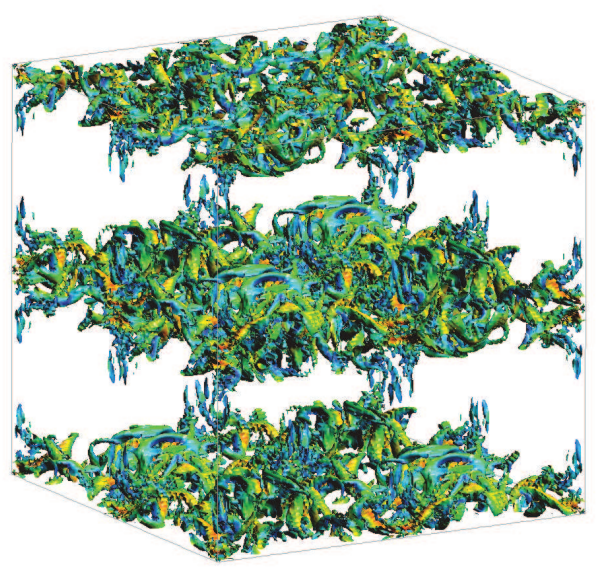

(f) Isosurface for $|\omega|=9.0$ at $t=10.2$

Figure 11: Vorticity isosurfaces with velocity contour at different time steps 


\subsubsection{Comparison of VMS methods}

In order to compare the different VMS methods defined previously and to test their performance as LES models we solve the TGV test on a $32^{3}$ linear elements mesh with a Reynolds number Re $=1,600$, for all the cases listed in Table 1. As for the DHIT test, the linear and static ASGS case (Sta-LinASGS) and the dynamic and linear ASGS case (Dyn-Lin-ASGS) diverge at some time step before $t=9$. Anyway, all the methods converge as $h \rightarrow 0$. We want to show the amount of numerical dissipation, the energy cascade in the spectra and the enstrophy evolution (compared to DNS) in all cases. We compare first the kinetic energy evolution with the kinetic energy evolution obtained by Brachet et al. [13], (Fig. $12(\mathrm{a}))$. We also present the energy spectra at $t=9$, when the flow is supposed to be nearly isotropic at large wave numbers, (Fig. 12(b)).

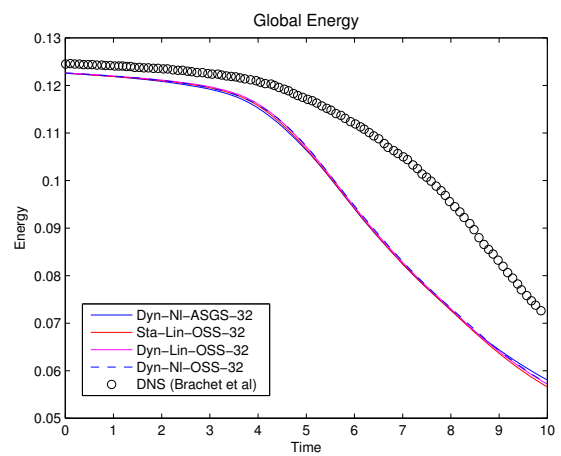

(a) Total kinetic energy evolution

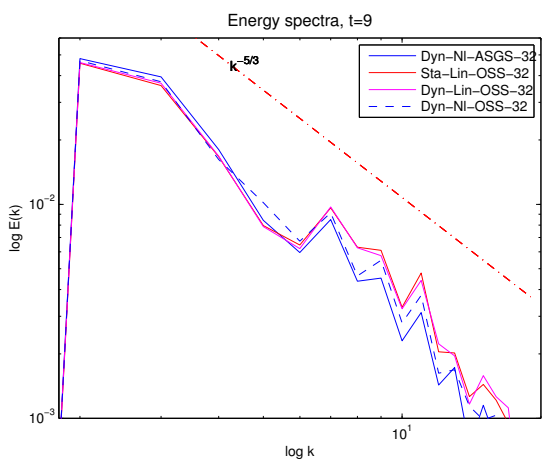

(b) Energy spectra at $t=9.0$

Figure 12: Total kinetic energy evolution and energy spectra

In Fig. 12(a), we can see that for a $32^{3}$ trilinear hexahedral elements mesh all methods show a premature decay of energy. We recognize the same behavior observed for the same mesh in the DHIT test, see Subsection 5.2.3. For this mesh, it is clear that the methods are not able to simulate properly the transition to turbulence. The energy spectra at $t=9.0$ shows us that the flow is isotropic at large wave numbers since it is decaying following the $k^{-5 / 3}$ Kolmogorov law.

As in the DHIT test, the cases with nonlinear and dynamic definitions of the subscales, using either ASGS or OSS methods, seem to be slightly less dissipative. Furthermore, OSS is a little bit less dissipative than ASGS, but the differences are not important.

\subsection{3. h-p refinement}

As in the DHIT problem, we perform a refinement study reducing $h$ and/or increasing $p$ using Dyn-Nl-OSS. Fig. 13(a) displays the total kinetic energy evolution for all the cases defined in Table 2, compared with the DNS [13]. The results show that all cases, excluding the $32^{3}$ linear hexahedral mesh, follow almost perfectly the line defined by the DNS result points.

In Fig. 13(b) we show the dissipation rate of the problem, also compared with the DNS results. The dissipation rate is directly related to the enstrophy of the problem, $\epsilon=2 \nu\left(\frac{1}{2}\left\langle|\omega|^{2}\right\rangle\right)$, where $|\omega|$ is the modulus of the vorticity. This parameter gives us an idea of how and when the flow is dissipating energy due to the viscous effects. The dissipation should have a peak when the energy is decaying faster and its shape is related to the slope in which the energy decays.

As in the DHIT problem three different groups of curves (excluding the DNS) can be observed in Fig. 13(b). First, the lowest curve, corresponding to the $32^{3}(Q 1)$ mesh, has a peak at $t \approx 7$, different from all the other curves. This means that the kinetic energy is decaying faster at this time for this case than for the remaining cases, which is in agreement with the results shown in Fig. 13(a). A second group of curves is made by those cases with $64^{3}$ discretization nodes, namely $64^{3}(Q 1)$ and $32^{3}(Q 2)$. These cases have a peak at $t \approx 8$, which is closer to the peak of the DNS. On the other hand, the shape of these curves is more similar to the DNS, since the energy evolution in Fig. 13(a) is also closer to the DNS one. We also have to note that $Q 2$ FEs perform better than $Q 1$ FEs for identical number of nodes. Finally, we distinguish a third group composed by the cases with $128^{3}$ nodes, which have a peak 


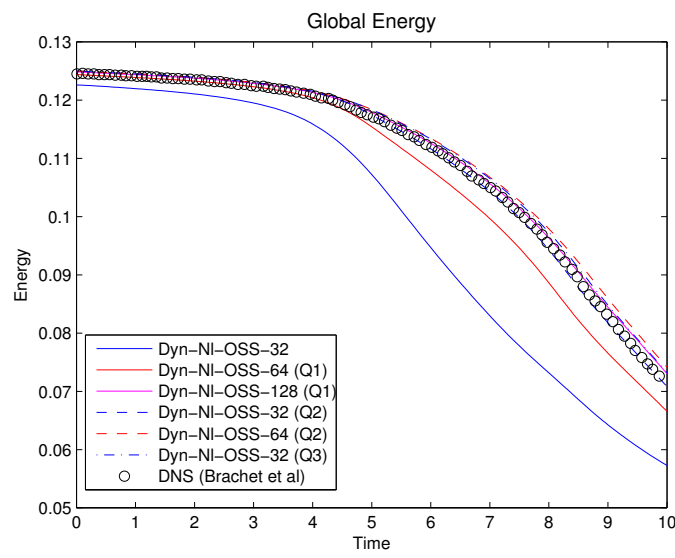

(a) Total kinetic energy evolution

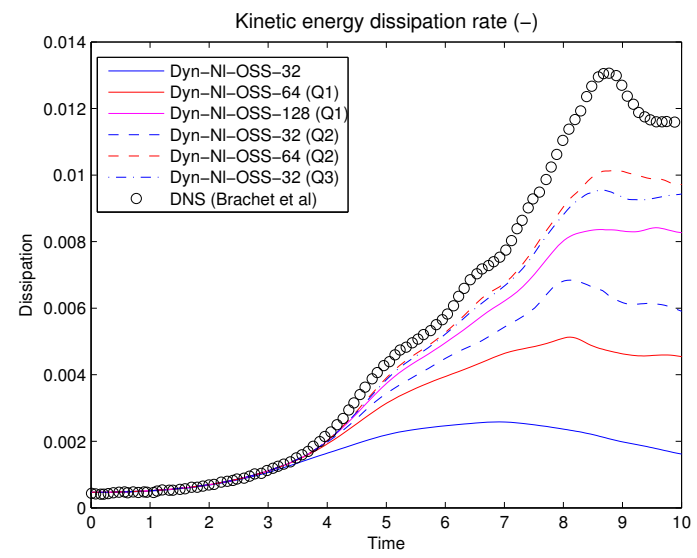

(b) Dissipation rate evolution

Figure 13: Total kinetic energy and dissipation rate evolution for the $h-p$ refinement cases

at about the same time than the DNS, $t \approx 9$. Among this group the $64^{3}(Q 2)$ discretization is the case that presents the best results compared with the DNS, as it was for the DHIT problem.

Remark 6.1. We have to note that all the quantities shown so far (kinetic energy, energy spectra and enstrophy) have been calculated only taking into account the resolved scales of the flow, that is, using the FE portion of the velocity, $\mathbf{u}_{h}$, instead the full velocity field, $\mathbf{u}$. As discussed in [22] we do not see any reason to consider $\mathbf{u}_{h}+\tilde{\mathbf{u}}$ a better solution than $\mathbf{u}_{h}$ once optimal convergence has been shown.

Fauconnier et al. [25] show separately the dissipation results obtained with the resolved scales and the total dissipation, taking into account the resolved and the subgrid scales. Our results for the cases with $64^{3}$ grid nodes, which are the used on [25], are, qualitatively speaking, similar to those obtained by the different methods proposed on that article only taking into account the resolved scales.

Fig. 14 displays the energy spectra at $t=9$, when the dissipation is maximum and the flow is evolving to turbulence. We compare the energy spectra obtained solving all the cases considered before with the DNS computed by [28], using the same Reynolds number $(\operatorname{Re}=1600)$ at the same time.

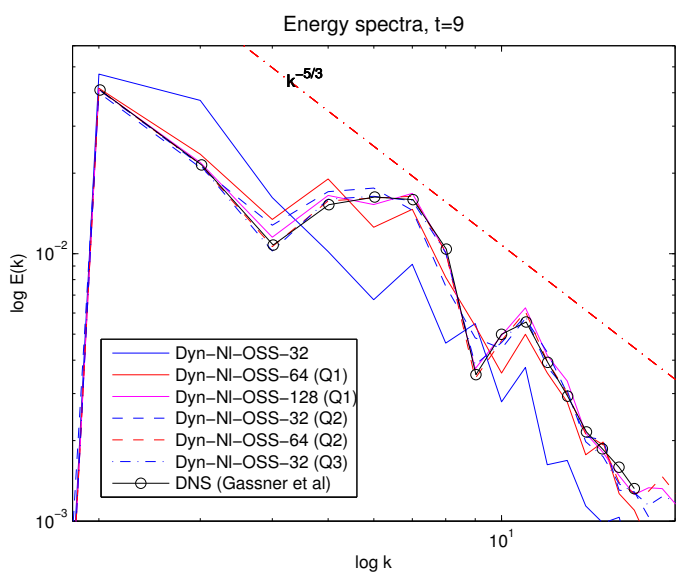

Figure 14: Energy spectra at $t=9$

Excluding the too coarse $32^{3}(Q 1)$ approximation, the energy spectra follow accurately the DNS energy spectra. For low wave numbers, the differences with the DNS are minimal. It is on the highest wave numbers where some differences between the methods appear. Note that the $32^{3}(Q 3)$ case follows 
very accurately the DNS spectrum. In general, we see that the spectra decay following a slope close to the $k^{-5 / 3}$ Kolmogorov law.

\section{Turbulent channel flow}

After studying the performance of VMS in the LES of homogenous flows we turn our attention to wall-bounded turbulent flow and present results of fully developed turbulent flow in a channel.

\subsection{Problem definition}

This test consists of a fluid that flows between two parallel walls driven by an imposed pressure gradient which is defined by the Reynolds number based on the wall shear velocity, $\operatorname{Re}_{\tau}$. In the important amount of literature devoted to this problem the usual Reynolds numbers are: $\operatorname{Re}_{\tau}=590, \operatorname{Re}_{\tau}=395$ and $\operatorname{Re}_{\tau}=180$ (see $[10,15,27,29,32,40,42,43,47,48]$ ). We will restrict our attention to $\operatorname{Re}_{\tau}=180$ and $\operatorname{Re}_{\tau}=395$.

\subsubsection{Computational domain and mesh definition}

We consider a computational domain defined by a box of length $\left(L_{x} \times L_{y} \times L_{z}\right)$, which depends on the Reynolds number. For $\operatorname{Re}_{\tau}=180$ the domain size is $(4 \pi \delta \times 2 \delta \times 4 / 3 \pi \delta)$ while for $\operatorname{Re}_{\tau}=395$ the size of the channel is $(2 \pi \delta \times 2 \delta \times 2 / 3 \pi \delta)$. The $x$-direction is the flow direction, also called streamwise direction, the $y$-direction is the wall-normal direction and the $z$-direction is the spanwise direction. In Fig. 15 we show schematically the geometry of the computational domain for this test.

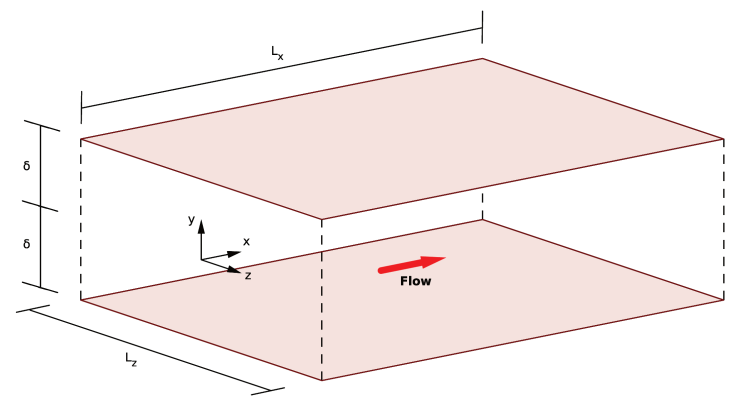

Figure 15: Channel computational domain

In the wall-normal direction boundaries $(y=-\delta$ and $y=\delta)$ we impose a non-slip condition. The streamwise and spanwise directions are assumed to be homogeneous, so we use periodic boundary conditions in these directions. ${ }^{3}$

We solve the problem using different mesh discretizations, all of them uniform in the homogeneous directions and refined near the walls in the wall-normal direction. In particular we will use meshes composed by $32^{3}, 64^{3}$ or $128^{3}$ hexahedral linear $(Q 1)$, quadratic $(Q 2)$ or cubic $(Q 3)$ elements. The refinement in the wall-normal direction follows a hyperbolic function, also used in [15, 27, 29, 32, 47], defined as

$$
y_{i}=\frac{\tanh \left(\gamma\left(\frac{2 i}{n p_{y}}-1\right)\right)}{\tanh (\gamma)},
$$

where $i=1, \ldots, n p_{y}$ with $n p_{y}$ the total amount of nodes in the wall-normal direction. Here, $\gamma$ is chosen to be equal to 2.75 for both $\operatorname{Re}_{\tau}=180$ and $\operatorname{Re}_{\tau}=395$.

\footnotetext{
${ }^{3}$ According to Kim et al. [43], the use of periodic boundary conditions in the homogeneous directions can be justified when the computational box is such that the largest eddies in the flow fit in the computational domain. This is the case considered here.
} 


\subsubsection{Setting}

As it has been said above, we solve the problem using two different friction Reynolds numbers, $\operatorname{Re}_{\tau}=180$ and $\operatorname{Re}_{\tau}=395$. We compare our results against those obtained by DNS in [48, 43] and we choose our parameters accordingly. We take the bulk mean velocity and the half channel height equal to one, $\bar{U}=1$ and $\delta=1$. The viscosity is computed from the estimated Reynolds number based on the bulk mean velocity $R e$. Then, from the friction Reynolds number $\operatorname{Re}_{\tau}$ we compute the friction velocity $(u \tau)$, the wall shear stress $\left(\tau_{w}\right)$ and a driving force equivalent to a pressure gradient $\left(f_{x}\right)$, given by [50]:

$$
u_{\tau}=\frac{\nu \operatorname{Re}_{\tau}}{\delta}, \quad \tau_{w}=\rho u_{\tau}^{2}, \quad f_{x}=\frac{\tau_{w}}{\delta} .
$$

We use the Crank-Nicolson time integration scheme with a constant time step. Ham et al. test in [33] the influence of the time step for a fully implicit Finite Difference midpoint method, equivalent to Crank-Nicolson, on the statistics of a channel flow DNS. They found little variation in statistical turbulence quantities up to $\delta t^{+}=1.6$. Following Gravemeier et al. [29], we define a time step in wall units $\delta t^{+}=\frac{\delta t u_{\tau}^{2}}{\nu} \approx 0.69$, which, according to [33], should not affect the turbulent quantity statistics. The same authors performed 25,000 time steps in order to allow the flow to develop and they collected the statistics during another 5,000 time steps. A total averaging time about $500 \delta / U_{0}$ is used in [17] once the statistically stable regime is achieved.

In Table 3 we present the value of the different parameters defined above for the two different friction Reynolds numbers.

\begin{tabular}{ccc}
\hline $\operatorname{Re}_{\tau}$ & 180 & 395 \\
\hline$\nu$ & $3.5714 \cdot 10^{-4}$ & $1.4545 \cdot 10^{-4}$ \\
$u_{\tau}$ & $6.4286 \cdot 10^{-2}$ & $5.7455 \cdot 10^{-2}$ \\
$\tau_{w}$ & $4.1327 \cdot 10^{-3}$ & $3.3010 \cdot 10^{-3}$ \\
$f_{x}$ & $4.1327 \cdot 10^{-3}$ & $3.3010 \cdot 10^{-3}$ \\
$\delta t$ & 0.06 & 0.03 \\
\hline
\end{tabular}

Table 3: Test parameters for the different friction Reynolds number

For the initial condition we impose a parabolic profile obtained solving the stationary Stokes problem with the driving force and viscosity defined above. Additionally, with the aim to achieve a fully developed flow earlier, we introduce a perturbation with a maximum value of $10 \%$ the bulk velocity.

Our purpose is to check the VMS methods defined in Subsection 2.2 for a wall-bounded flow. Following the computations performed for the previous tests, we solve the problem using the same cases defined in Table 1 and the numerical parameters $\tau_{c}=0$ and $\tau_{m}$ are defined in the same way, now with the algorithmic constants $c_{1}=12$ and $c_{2}=8$ (see Subsection 8) and the characteristic length, $h$, is choosen to be the minumum element length.

\subsection{Numerical results}

\subsubsection{Velocity profiles}

We first present the mean streamwise velocity profile scaled by the wall shear stress velocity, $\langle u\rangle^{+}=$ $\frac{\langle u\rangle}{u_{\tau}}$ for all cases defined in Table 1 , where $\langle\cdot\rangle$ denotes the mean value in streamwise and spanwise direction and in time.

In Fig. 16(a) we show the mean streamwise velocity normalized by the wall-shear velocity, $u_{\tau}$, obtained for all cases considered in Table 1 in a $32^{3}$ trilinear elements mesh for the $\operatorname{Re}_{\tau}=395$ case. We compare the results with the DNS one obtained in [48]. We can observe in Fig. 16(a) that all methods perform quite similar and fit pretty well to the DNS result. Fig. 16 also depicts the streamwise, spanwise and wall-normal root mean square (rms) velocity fluctuation components normalized by the wall-shear stress velocity. 


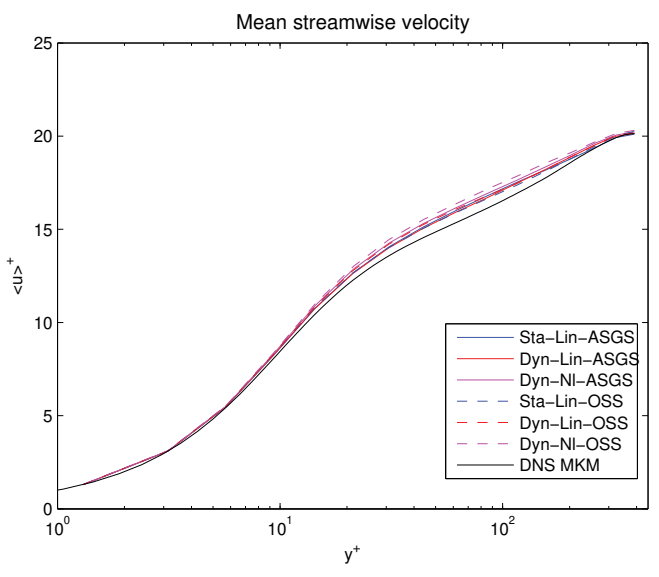

(a) Mean streamwise velocity

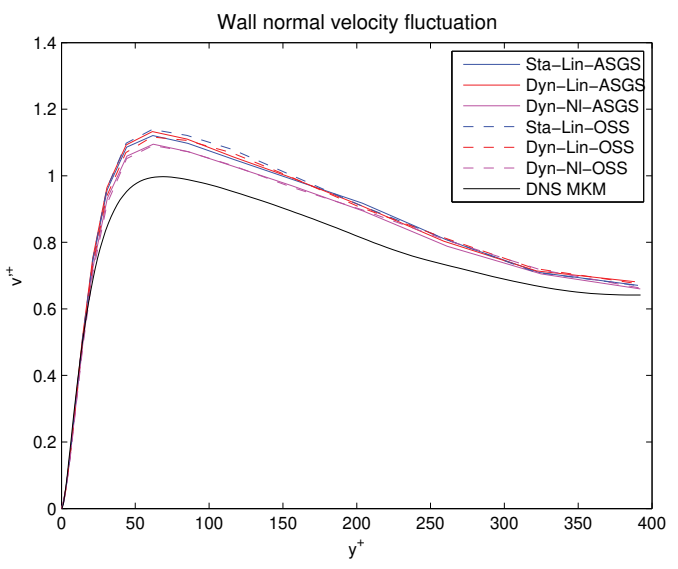

(c) Rms wall-normal velocity fluctuation

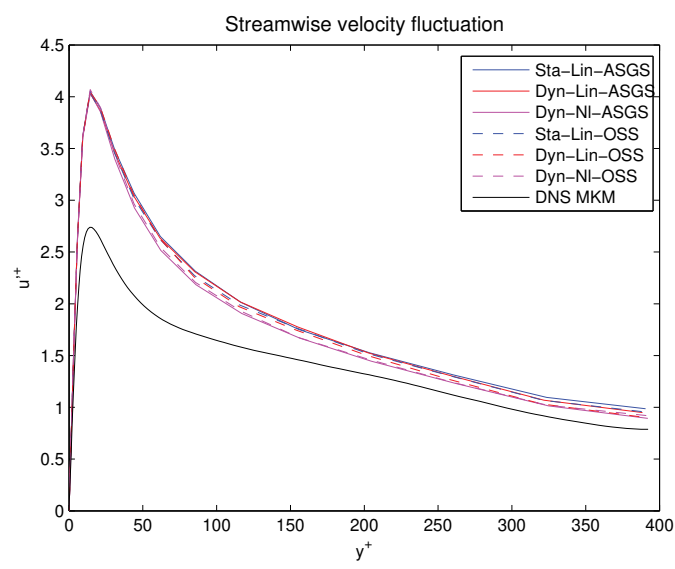

(b) Rms streamwise velocity fluctuation

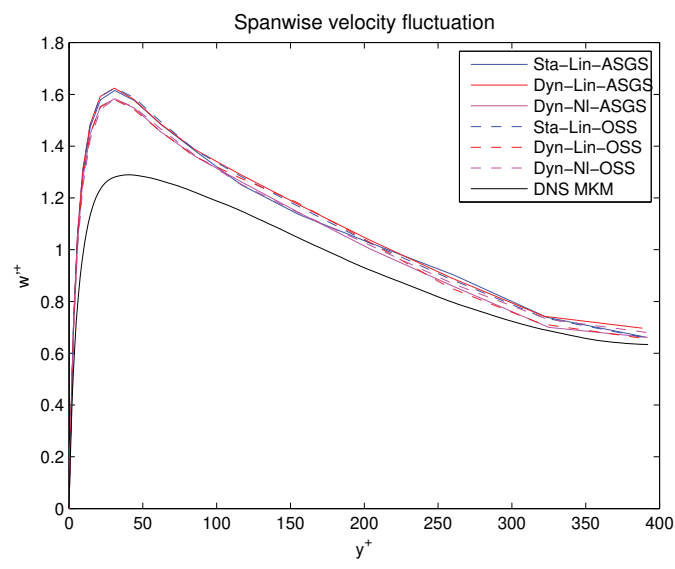

(d) Rms spanwise velocity fluctuation

Figure 16: Mean streamwise velocity and rms velocity fluctuations for $\operatorname{Re}_{\tau}=395$ case using a $32^{3} Q 1$ mesh

\subsubsection{Reynolds shear stress}

Another turbulent quantity widely used in the channel flow test is the Reynolds shear stress. At the continuous level the Reynolds shear stress is defined as

$$
R_{x y}=-\left\langle u^{\prime} v^{\prime}\right\rangle+\nu \frac{\partial\langle u\rangle}{\partial y}
$$

being $u$ and $v$ the velocity in the streamwise direction and wall-normal direction, respectively, and the prime denoting the fluctuations, that is to say, the variable minus the mean.

It can be seen that for the discrete equation (13), one can obtain the Reynolds shear stress defined as follows:

$$
R_{x y}=-\left\langle a_{x}^{\prime} a_{y}^{\prime}\right\rangle+\nu \frac{\partial\left\langle u_{h}\right\rangle}{\partial y}=-\underbrace{\left\langle u_{h}^{\prime} v_{h}^{\prime}\right\rangle}_{I}-\underbrace{\left\langle u_{h}^{\prime} \tilde{v}^{\prime}\right\rangle-\left\langle\tilde{u}^{\prime} v_{h}^{\prime}\right\rangle-\left\langle\tilde{u}^{\prime} \tilde{v}^{\prime}\right\rangle}_{I I}+\underbrace{\nu \frac{\partial\left\langle u_{h}\right\rangle}{\partial y}}_{I I I} .
$$

being $a_{i}$ the $i$ component of the advection velocity. In (59) we have used the nonlinear definition of the advection velocity defined in (25).

The first term on the second part of (59) (term $I$ ) is the contribution of the resolved scales, the FE component, to the cross term $\left\langle a_{x}^{\prime} a_{y}^{\prime}\right\rangle$. Term II denotes the contribution of the subgrid scales and their interaction with the FE components, that is the unresolved part of the equation. Finally, term 
III accounts for the viscous portion of the Reynolds shear stress. Note that the derivatives of the approximated subscales are not computable, since these approximated subscales are discontinuous and have been designed to approximate the effect of the exact subscales on the finite element scales on the element.

For a fully developed and statistically stable turbulent flow, the Reynolds shear stress along the wallnormal direction has a linear shape (see [43]). Normalized by the viscous term $I I I$ value at the wall, the total Reynolds shear stress in terms of $y / \delta$ should have the following expression: $R_{x y}(y / \delta)=(-y / \delta)$. Fig. 17 depicts the absolute value of the Reynolds shear stress along the upper half channel $(y>0)$, with the different terms appearing in (59) and compared with the DNS in [48], for the Dyn-Nl-OSS case with $\operatorname{Re}_{\tau}=395$. The computed results are almost identical to the DNS ones. It has to be noted that the computed results are evaluated at the integration points due to the presence of the derivative in the Reynolds shear stress, which using linear FEs is constant in each element. Then, using two Gauss points per direction for the numerical integration, term III will be constant for those two Gauss points being at the same element. This behavior is observed in Fig. 17, where the viscous term is pairwise constant. This last fact also affects the total Reynolds shear stress, since the resolved term has different values at each element Gauss point, the sum of terms $I$ and $I I I$ results in an oscillatory shape near the wall, where the viscous term is more relevant. It is also seen that the unresolved term II does not contribute to the Reynolds shear stress, which is a good property of the tested VMS methods. The results for the remaining cases in Table 1 are similar to those presented in Fig. 17 and have not been reported.

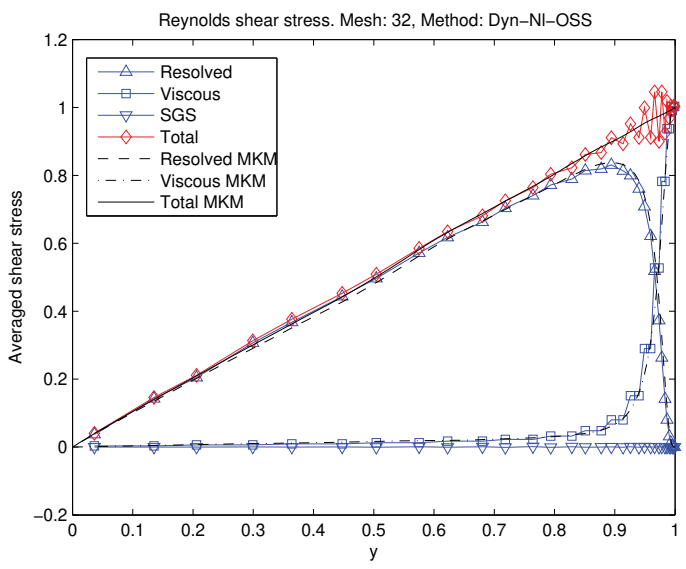

Figure 17: Reynolds stress of the Dyn-Nl-OSS case

\section{Sensitivity with respect to the stabilization parameters}

All the VMS models considered herein depend on the stabilization parameters $\tau_{m}$ and $\tau_{c}$, which contain constants $c_{1}$ and $c_{2}$ whose value is chosen from numerical experiments. However, we can infer from (38) or (39) how this dependency will be. As mentioned before, the last two terms in (39) are dissipative and therefore increasing $\tau_{m}$ and/or $\tau_{c}$ we obtain a more dissipative method. From (20)-(21), increasing $\tau_{m}$ results in a reduction of $\tau_{c}$. More precisely

$$
\tau_{c}=\nu+\frac{c_{2}}{c_{1}}|\mathbf{a}| h
$$

from where we see that increasing $c_{1}$ reduces both $\tau_{m}$ and $\tau_{c}$ but increasing $c_{2}$ reduces $\tau_{m}$ but increases $\tau_{c}$. On the other hand, only the fourth term in (39) is essential to control $\tau_{m}\left\|\mathcal{P}\left(\mathbf{a} \cdot \nabla \mathbf{u}_{h}+\nabla p_{h}\right)\right\|^{2}$ and it is possible to choose $\tau_{c}=0$.

The results presented above have been obtained using different settings of the numerical stabilization parameters $\tau_{m}$ and $\tau_{c}$. In DHIT and TGV tests, we take the algorithmic constants $c_{1}=12$ and $c_{2}=2$ for $\tau_{m}$ and we set $\tau_{c}=0$, while for the TCF test we have used $c_{1}=12$ and $c_{2}=8$ for $\tau_{m}$ and also 
$\tau_{c}=0$. In this section we analyze the influence of these parameters on the numerical results and justify our choice of the constants for the large eddy simulation of turbulent flows.

We have performed a sensitivity analysis of the VMS schemes with respect to the value of $c_{1}$ and $c_{2}$. To see the effect of such algorithmic constants on $\tau_{m}$ and $\tau_{c}$ independently, we define a new constant $c_{c}$ which allow us to redefine (60) as

$$
\tau_{c}=c_{c}\left(\nu+\frac{c_{2}}{c_{1}}|\mathbf{a}| h\right)
$$

These experiments have been done for the DHIT test using the Dyn-Nl-OSS case in a $32^{3} Q 1$ mesh and the results are depicted in Fig. 20. They show important changes in the dissipation the VMS methods introduce when constants are changed. In particular, for high Reynolds number problems, the constant $c_{1}$ does not have so much influence on $\tau_{m}$, as it can be seen in Fig. 20(b) where $c_{c}=0$, but it does on $\tau_{c}$ (when $c_{c}=1$ in (61)). With respect to $c_{2}$, we observe in Fig. 20(b) that it influences the energy dissipation of the method, which is increased when the value of this constant is decreased. When $\tau_{c}$ is activated $\left(c_{c}=1\right)$, we observe a growth of the energy dissipation when the coefficient $c_{2} / c_{1}$ increases, see Fig. 20(e). This behavior is what we are expecting since the method becomes more diffusive when $\tau_{c}$ is increased due to the last term in (39).

Concerning the energy spectra, it is also shown in Fig. 20(c) and Fig. 20(d) that the only constant that influences the result when $\tau_{c}=0$ is $c_{2}$. In these figures we can see that when we increase $c_{2}$ the method is less dissipative, resulting in an inappropriate slope of the energy spectra. We can observe that with $c_{2}=2$ the decay of the energy behaves correctly, keeping the $k^{-5 / 3}$ law. For the largest values of $c_{2}$ the energy at small scales is not properly dissipated. Note that for $c_{2}=2$ the slope of the energy spectrum is kept almost constant along the time, which does not happen in the other cases. When we activate $\tau_{c}$, Figures $20(\mathrm{f})$ and $20(\mathrm{~g})$, we are introducing additional dissipation into the system which eliminates the pile up of the energy spectra for all the cases, but generally results in steeper slopes. Here we also have to note that the energy spectra slope is time dependent for all cases except for $c_{2}=2$. This analysis led us to choose $c_{1}=12$ and $c_{2}=2$ for $\tau_{m}$ and set $\tau_{c}=0$ for homogeneous turbulence, i.e., DHIT and TGV tests.

In order to go in depth about the effect of the algorithmic constants $c_{1}$ and $c_{2}$ and the stabilization parameter $\tau_{c}$ of the incompressibility equation, we compare the results for the TCF problem with a friction Reynolds number $\operatorname{Re}_{\tau}=180$ using the same choice made for homogeneous turbulence $\left(c_{1}=12\right.$, $c_{2}=2$ and $\left.\tau_{c}=0\right)$ against the setting of the incompressible case in [3] $\left(c_{1}=12, c_{2}=2\right.$ and $\tau_{c}$ as in (21)) and a less dissipative setting with $c_{1}=12, c_{2}=8$ and $\tau_{c}=0$.

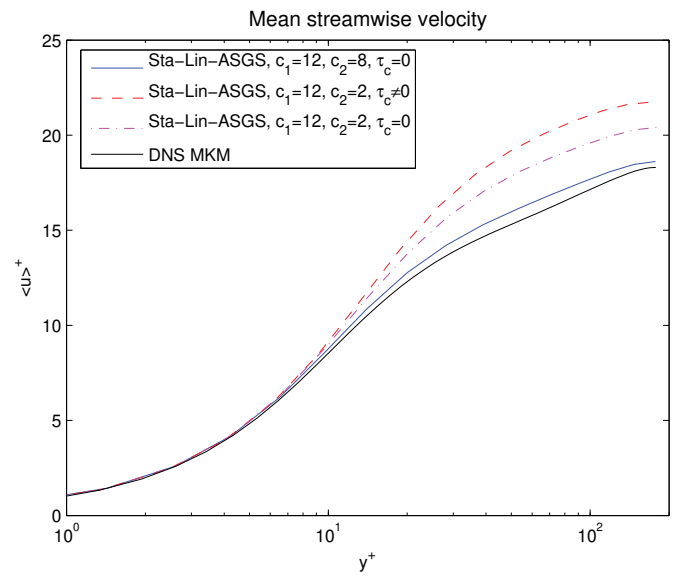

Figure 18: Comparison of mean streamwise velocity for $\operatorname{Re}_{\tau}=180$ case using a $32^{3} Q 1$ mesh

In Fig. 18 the mean velocity in the streamwise direction is shown. As in the case of homogeneous turbulence, some differences between the three cases can be observed, the choice used in section 7 being the most accurate one. The effect of the algorithmic constant $c_{2}$ and the stabilization parameter $\tau_{c}$ in the problem solution can be clearly observed, i.e., the less dissipative choice gives the best results. Fig. 


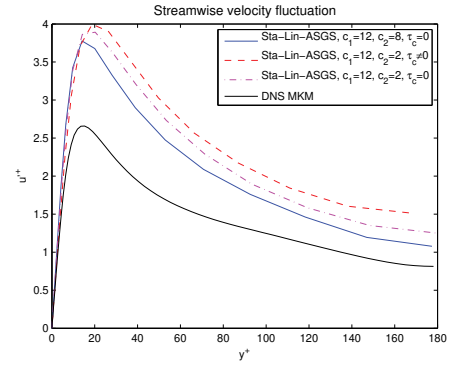

(a) Rms streamwise velocity fluctua- (b) Rms wall-normal velocity fluctuation tion
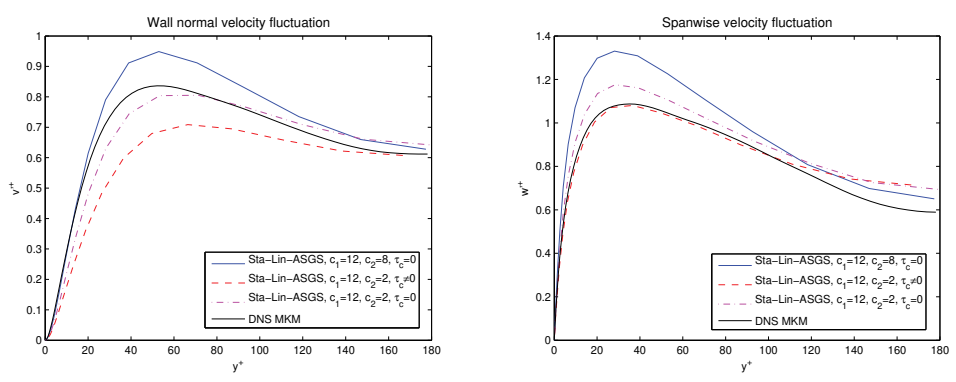

(c) Rms spanwise velocity fluctuation

Figure 19: Comparison of rms velocity fluctuations 


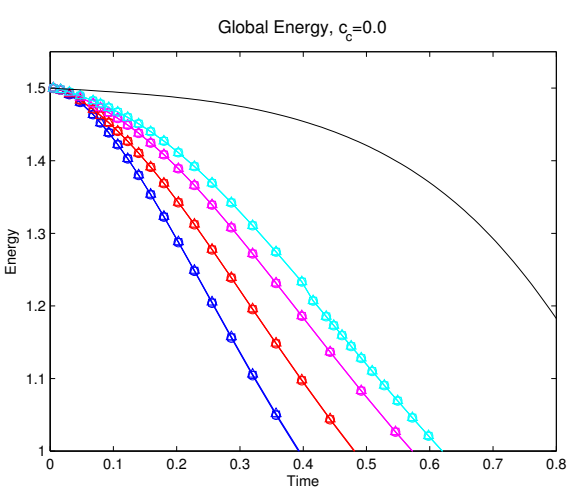

(b) Global energy with $c_{c}=0$

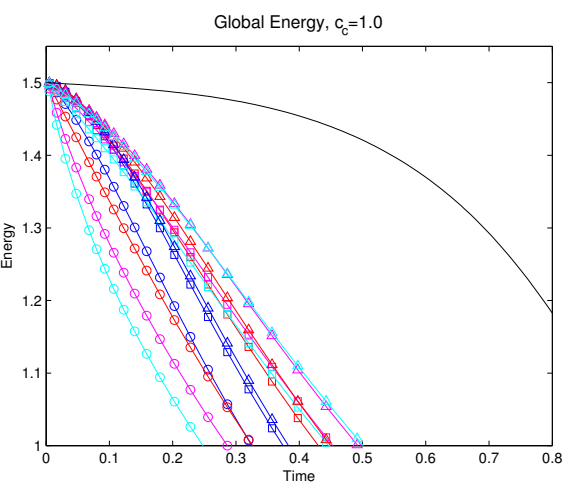

(e) Global energy with $c_{c}=1$

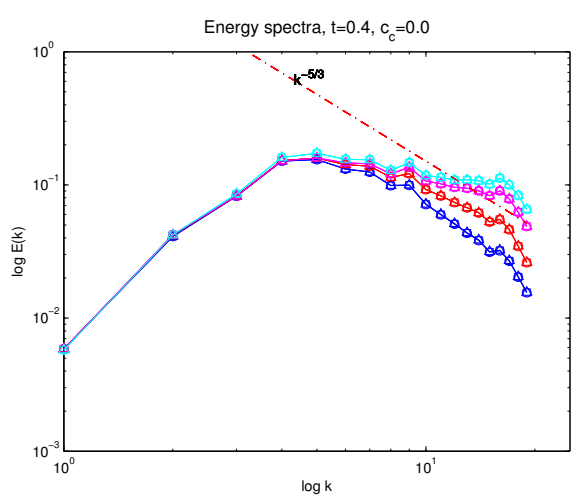

(c) Energy spectra at $t=0.4$ with $c_{c}=0$

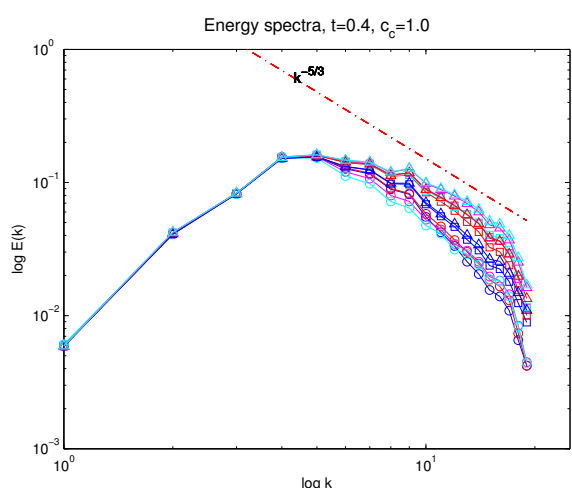

(f) Energy spectra at $t=0.4$ with $c_{c}=1$

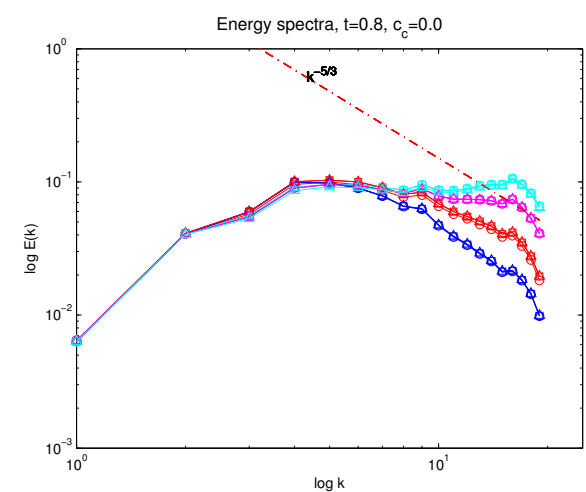

(d) Energy spectra at $t=0.8$ with $c_{c}=0$

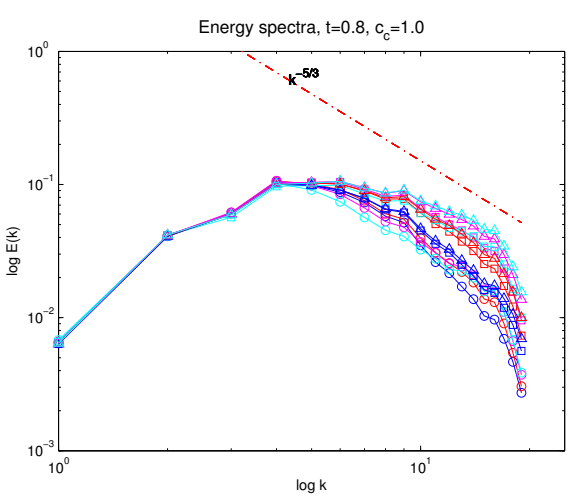

(g) Energy spectra at $t=0.8$ with $c_{c}=1$

Figure 20: Comparison of the global energy and energy spectra for different $c_{1}, c_{2}$ and $c_{c}$ in the DHIT test 
19 depicts the rms velocity fluctuations in all directions. The fluctuations in the streamwise direction are better predicted using $\left(c_{1}=12, c_{2}=8\right.$ and $\left.\tau_{c}=0\right)$ but the spanwise and wall-normal directions are not.

\section{Behavior in the small time step limit}

Small time step instabilities for VMS LES simulations of turbulent flows have been reported in $[34,27]$. In these references, the VMS models differ from the ones in this work. Instead of the definition of $\tau_{m}$ in (20), a time step dependent stabilization parameter $\tau_{m}$

$$
\tau_{m}=\left(\frac{1}{\delta t}+\frac{c_{1} \nu}{h^{2}}+\frac{c_{2}|\mathbf{a}|}{h}\right)^{-1},
$$

is considered in all cases. ${ }^{4}$ The plain introduction of a time step dependency in $\tau_{m}$ faces serious difficulties:

- The method becomes unstable in the small time step limit since it converges to the unstable Galerkin formulation.

- If $\tau_{c}$ is computed from (21) (as it is usually done, see, e.g., [10, 34, 27, 29]), $\tau_{m} \sim \delta t$ and $\tau_{c} \sim \delta t^{-1}$ in the small time step limit. If this approach is followed, the essential numerical dissipation given by the fifth term in (38) is reduced as $\delta t \rightarrow 0$, whereas the numerical dissipation introduced by the last term in the left hand side (a incompressibility penalty term) of (38) is increased. It has a compensating effect in practice, but the penalty term does not properly act as a turbulence model.

Let us perform a test to study the small time step behavior of the VMS methods presented in Section 2 , using the skew-symmetric type 1 form of the convective term, as in previous numerical experiments. We also include a combination we do not advocate here, static subscales and nonlinear splitting, an approach followed in $[16,10,34,27,29]$. The behaviour of all the methods for the TCF test with $\delta t=0.002$ is summarized in Table 4 , where YES means that the simulation was succesful, NO means that the simulation diverged and $\delta t \downarrow$ means that the simulation was succesful only when the adaptive time step strategy described in Section 5.1.2 was used.

It is important to note that the static and nonlinear ASGS formulation used in $[10,34,27,29]$ with the convective term type 2 becomes unstable after some time, as also reported in these works, even for the time step size defined in section 7.1.2. However, using the the skew-symmetric type 1 form of the convective term, which exactly conserves energy, the simulation ended succesfully for the time step defined in section 7.1.2, but failed to converge with the small one. This result is a numerical evidence of the fact that the use of convective terms without the skew-symmetric property produce energy (see also Section 5.2.3) that can make simulations unstable. Further, these results evidence once again that it is a good choice to stick to provably unconditionally stable formulations, i.e., the dynamic formulations and/or orthogonal subscales formulations with a skew-symmetric convective term.

\begin{tabular}{ccccccccc}
\hline Method & \multicolumn{4}{c}{ ASGS } & \multicolumn{3}{c}{ OSS } \\
\hline Tracking & \multicolumn{2}{c}{ Static } & \multicolumn{2}{c}{ Dynamic } & \multicolumn{2}{c}{ Static } & \multicolumn{2}{c}{ Dynamic } \\
\hline Advection & Linear & Nonlinear & Linear & Nonlinear & Linear & Nonlinear & Linear & Nonlinear \\
\hline Converged & Yes & No & Yes & Yes & $\delta t \downarrow$ & $\delta t \downarrow$ & Yes & Yes \\
\hline
\end{tabular}

Table 4: Small time step convergence analysis

\footnotetext{
${ }^{4}$ The parameter $\tau_{m, t}$ for the dynamic subscales model also scales with $\delta t$, as discussed in Section (4). However, this dependence comes from a consistent time integration of the subscale time derivative (see also [22, Section 3.2]).
} 


\section{Conclusions}

In this paper we have assessed the performance of the numerical formulations previously developed in our group [19, 22, 21, 52] for turbulent incompressible flow problems. The methods proposed are different to those whose testing in turbulent regimes has been published before, the closest ones being those reported in $[10,27]$. In [10] the ASGS method with quasi-static subscales is used but the time step dependency is included in the stabilization parameter (with the inconsistencies and problems discussed in section 9) and the nonlinear scale splitting is applied in the finite element equation only (not in the subscale equation). Time dependendent subscales are used in [27], but the authors consider a linear scale splitting. Furthermore, in both works $\tau_{c} \neq 0$.

Apart from the numerical experimentation, we have also discussed some theoretical aspects, such as the dissipative structure of the methods and the way energy is conserved, which we have numerically verified. Related to this point, one of the aspects treated is the use of different skew-symmetric forms of the convective term, with its impact on energy conservation and the realization that if a skew symmetric form is not used negative energy dissipation may introduced to the scheme, which may be a source of instability. Other side points that we have discussed and checked numerically are the dependence of the algorithmic constants on the results or the effect of small time steps when the stabilization parameters depend on them.

The most important conclusions however come from the different problems that we have solved numerically. As a general conclusion, all methods presented yield similar results, all displaying the features of turbulent flows, reproducing appropriately global outputs such as energy spectra. The methods are stable and converge to reference solutions, both when the mesh is refined and when the polynomial order is increased. Regarding this point, let us mention that we have found a good compromise to use quadratic elements, with better results than for linear elements with the same number of degrees of freedom. Likewise, the OSS method with dynamic subscales could be the formulation to be favored, since it is the one that introduces (slightly) less dissipation and shows better results in the time evolution of global energy, completely free of oscillations.

On the other hand, we have thoroughly analyzed the effect of the algorithmic constants for isotropic turbulence and wall-bounded turbulent flows, and chose them based on this sensitivity analysis. An important observation in this line is the fact that all the methods considered in this work are certainly sensitive to the algorithmic constants and they have to be properly chosen in order to simulate turbulent flows. In fact, the difference in the numerical results are much more influenced by the algorithmic constants than by the choice of the VMS formulation itself. This strong influence seems to be a characteristic feature of turbulence, since in our experience it is not so important in laminar flows.

Apart from the quality of the results, the OSS method with dynamic subscales is convenient in terms of numerical performance. It requires more nonlinear iterations than ASGS, but less iterations of the linear solver, altogether leading to lower computational cost. In both formulations, ASGS and OSS, the use of dynamic subscales has been found to be crucial for nonlinear convergence. In fact, in some cases quasi-static subscales have even failed to converge.

\section{Acknowledgements}

This work has been partially funded by the European Research Council under the FP7 Programme Ideas through the Starting Grant No. 258443 - COMFUS: Computational Methods for Fusion Technology. We also acknowledge the financial support received from the Spanish Ministry of Economics and Competitiveness, National Programme of R\&D to the project PARANAT (ENE2011-28825). R. Codina gratefully acknowledges the support received from the ICREA Acadèmia Progam from the Catalan Government. Furthermore, O. Colomés would like to acknowledge the support received from the Catalan Government through a FI fellowship.

[1] AGARD-AR-345. A selection of test cases for the validation of large-eddy simulations of turbulent flows. Technical report, 1998.

[2] M. Avila, J. Principe, and R. Codina. A finite element dynamical nonlinear subscale approximation for the low Mach number flow equations. Journal of Computational Physics, 230(10-11):7988-8009, 2011. 
[3] M. Avila, J. Principe, and R. Codina. Large eddy simulation of low Mach number flows using a dynamical and nonlinear finite element subgrid scale model. submitted.

[4] S. Badia. On stabilized finite element methods based on the Scott-Zhang projector. Circumventing the inf-sup condition for the Stokes problem. Computer Methods in Applied Mechanics and Engineering, 247-248(0):65-72, 2012.

[5] S. Badia and R. Codina. On a multiscale approach to the transient Stokes problem: Dynamic subscales and anisotropic space-time discretization. Applied Mathematics and Computation, 207(2):415-433, 2009.

[6] S. Badia, R. Codina, and J. V. Gutiérrez-Santacreu. Long-term stability estimates and existence of a global attractor in a finite element approximation of the Navier-Stokes equations with numerical subgrid scale modeling. SIAM J. Numer. Anal., 48(3):1013-1037, 2010.

[7] S. Badia and J. V. Gutiérrez-Santacreu. Convergence towards weak solutions of the Navier-Stokes equations for a finite element approximation with numerical subgrid scale modeling. IMA Journal of Numerical Analysis, In press, 2013.

[8] S. Badia, A. Martín, and J.Principe. Enhanced balancing Neumann-Neumann preconditioning in computational fluid and solid mechanics. International Journal for Numerical Methods in Engineering, 96(4):203-230, 2013.

[9] S. Badia, A. F. Martín, and J. Principe. Implementation and scalability analysis of balancing domain decomposition methods. Archives of Computer Methods in Engineering, 20:239-262, 2013.

[10] Y. Bazilevs, V. Calo, J. Cottrell, T. Hughes, A. Reali, and G. Scovazzi. Variational multiscale residual-based turbulence modeling for large eddy simulation of incompressible flows. Computer Methods in Applied Mechanics and Engineering, 197(1-4):173-201, 2007.

[11] A. D. Beck and G. J. Gassner. Numerical simulation of the Taylor-Green vortex at $R e=1600$ with the Discontinuous Galerkin Spectral Element method for well-resolved and underresolved scenarios. 1st International Workshop on High-Order CFD Methods at the 50th AIAA Aerospace Sciences Meeting, Nashville, TN, 2012.

[12] J. P. Boris, F. F. Grinstein, E. S. Oran, and R. L. Kolbe. New insights into large eddy simulation. Fluid Dynamics Research, 10(4-6):199, 1992.

[13] M. E. Brachet, D. I. Meiron, S. A. Orszag, B. G. Nickel, R. H. Morf, and U. Frisch. Small-scale structure of the Taylor-Green vortex. Journal of Fluid Mechanics, 130:411-452, 1983.

[14] A. N. Brooks and T. J. R. Hughes. Streamline upwind/Petrov-Galerkin formulations for convection dominated flows with particular emphasis on the incompresible Navier-Stokes equations. Computer Methods in Applied Mechanics and Engineering, 32:199-259, 1982.

[15] R. Calderer and A. Masud. Residual-based variational multiscale turbulence models for unstructured tetrahedral meshes. Computer Methods in Applied Mechanics and Engineering, 254(0):238253, 2013.

[16] V. Calo. Residual based mulstiscale turbulence modeling: finite volume simulations of bypass transition. PhD thesis, Department of Civil and Environmental Engineering, Stanford University, 2004.

[17] H. Choi and P. Moin. Effects of the computational time step on numerical solutions of turbulent flow. Journal of Computational Physics, 113(1):1-4, 1994.

[18] R. Codina. Stabilization of incompressibility and convection through orthogonal sub-scales in finite element methods. Computer Methods in Applied Mechanics and Engineering, 190(13-14):1579$1599,2000$. 
[19] R. Codina. Stabilized finite element approximation of transient incompressible flows using orthogonal subscales. Computer Methods in Applied Mechanics and Engineering, 191(39-40):4295-4321, 2002 .

[20] R. Codina. Analysis of a stabilized finite element approximation of the Oseen equations using orthogonal subscales. Applied Numerical Mathematics, 58:264-283, 2008.

[21] R. Codina, J. Principe, and S. Badia. Dissipative structure and long term behavior of a finite element approximation of incompressible flows with numerical subgrid scale modeling. In R. Borst and E. Ramm, editors, Multiscale Methods in Computational Mechanics, volume 55 of Lecture Notes in Applied and Computational Mechanics, pages 75-93. Springer Netherlands, 2011.

[22] R. Codina, J. Principe, O. Guasch, and S. Badia. Time dependent subscales in the stabilized finite element approximation of incompressible flow problems. Computer Methods in Applied Mechanics and Engineering, 196(21-24):2413-2430, 2007.

[23] C. R. Dohrmann. A preconditioner for substructuring based on constrained energy minimization. SIAM Journal on Scientific Computing, 25(1):246-258, 2003.

[24] J. Douglas and J. Wang. An absolutely stabilized finite element method for the Stokes problem. Mathematics of computation, 52:495-508, 1989.

[25] D. Fauconnier, C. De Langhe, and E. Dick. Construction of explicit and implicit dynamic finite difference schemes and application to the large-eddy simulation of the Taylor-Green vortex. Journal of Computational Physics, 228(21):8053-8084, 2009.

[26] C. Fureby and F. F. Grinstein. Large eddy simulation of high-Reynolds-number free and wallbounded flows. Journal of Computational Physics, 181(1):68-97, 2002.

[27] P. Gamnitzer, V. Gravemeier, and W. A. Wall. Time-dependent subgrid scales in residual-based large eddy simulation of turbulent channel flow. Computer Methods in Applied Mechanics and Engineering, 199(13-16):819-827, 2010.

[28] G. Gassner and A. Beck. On the accuracy of high-order discretizations for underresolved turbulence simulations. Theoretical and Computational Fluid Dynamics, pages 1-17.

[29] V. Gravemeier, M. W. Gee, M. Kronbichler, and W. A. Wall. An algebraic variational multiscalemultigrid method for large eddy simulation of turbulent flow. Computer Methods in Applied Mechanics and Engineering, 199(13-16):853-864, 2010.

[30] F. F. Grinstein, L. G. Margolin, and W. J. Rider. Implicit large eddy simulation: computing turbulent fluid dynamics. Cambridge university press, 2007.

[31] O. Guasch and R. Codina. Statistical behavior of the orthogonal subgrid scale stabilization terms in the finite element large eddy simulation of turbulent flows. Computer Methods in Applied Mechanics and Engineering, 2013.

[32] J. Gullbrand and F. K. Chow. The effect of numerical errors and turbulence models in largeeddy simulations of channel flow, with and without explicit filtering. Journal of Fluid Mechanics, 495:323-341, 2003.

[33] F. Ham, F. Lien, and A. Strong. A fully conservative second-order finite difference scheme for incompressible flow on nonuniform grids. Journal of Computational Physics, 177(1):117-133, 2002.

[34] M.-C. Hsu, Y. Bazilevs, V. Calo, T. Tezduyar, and T. Hughes. Improving stability of stabilized and multiscale formulations in flow simulations at small time steps. Computer Methods in Applied Mechanics and Engineering, 199(13-16):828 - 840, 2010.

[35] T. Hughes, J. Cottrell, and Y. Bazilevs. Isogeometric analysis: CAD, finite elements, NURBS, exact geometry and mesh refinement. Computer Methods in Applied Mechanics and Engineering, 194(39-41):4135 - 4195, 2005. 
[36] T. J. Hughes. Multiscale phenomena: Green's functions, the Dirichlet-to-Neumann formulation, subgrid scale models, bubbles and the origins of stabilized methods. Computer Methods in Applied Mechanics and Engineering, 127(1-4):387-401, 1995.

[37] T. J. Hughes, G. R. Feijóo, L. Mazzei, and J.-B. Quincy. The variational multiscale method - A paradigm for computational mechanics. Computer Methods in Applied Mechanics and Engineering, 166(1-2):3-24, 1998.

[38] T. J. R. Hughes, L. P. Franca, and M. Balestra. A new finite element formulation for computational fluid dynamics: V. Circumventing the Babuska-Brezzi condition: a stable Petrov-Galerkin formulation of the Stokes problem accommodating equal-order interpolations. Computer Methods in Applied Mechanics and Engineering, 59(1):85-99, 1986.

[39] T. J. R. Hughes, L. Mazzei, and K. E. Jansen. Large eddy simulation and the variational multiscale method. Computing and Visualization in Science, 3:47-59, 2000.

[40] T. J. R. Hughes, A. A. Oberai, and L. Mazzei. Large eddy simulation of turbulent channel flows by the variational multiscale method. Physics of Fluids, 13(6):1784-1799, 2001.

[41] J.B. Chapelier, M. De La Llave Plata, and F. Renac, E. Martin. Final abstract for ONERA Taylor-Green DG participation, 2012.

[42] V. John and A. Kindl. Variants of projection-based finite element variational multiscale methods for the simulation of turbulent flows. International Journal for Numerical Methods in Fluids, 56(8):1321-1328, 2008.

[43] J. Kim, P. Moin, and R. Moser. Turbulence statistics in fully developed channel flow at low Reynolds number. Journal of Fluid Mechanics, 177:133-166, 1987.

[44] B. Koobus and C. Farhat. A variational multiscale method for the large eddy simulation of compressible turbulent flows on unstructured meshes-application to vortex shedding. Computer Methods in Applied Mechanics and Engineering, 193(15-16):1367-1383, 2004.

[45] J. Mandel. Balancing domain decomposition. Communications in Numerical Methods in Engineering, 9(3):233-241, 1993.

[46] N. N. Mansour and A. A. Wray. Decay of isotropic turbulence at low Reynolds number. Physics of Fluids, 6(2):808-814, 1994.

[47] A. Masud and R. Calderer. A variational multiscale method for incompressible turbulent flows: Bubble functions and fine scale fields. Computer Methods in Applied Mechanics and Engineering, 200(33-36):2577-2593, 2011.

[48] R. D. Moser, J. Kim, and N. N. Mansour. Direct numerical simulation of turbulent channel flow up to $R e_{\tau}=590$. Physics of Fluids, 11(4):943-945, 1999.

[49] X. Nogueira, L. Cueto-Felgueroso, I. Colominas, and H. Gómez. Implicit large eddy simulation of non-wall-bounded turbulent flows based on the multiscale properties of a high-order finite volume method. Computer Methods in Applied Mechanics and Engineering, 199(912):615-624, Jan. 2010.

[50] S. B. Pope. Turbulent Flows. Cambridge University Press, 2000.

[51] J. Principe and R. Codina. On the stabilization parameter in the subgrid scale approximation of scalar convection-diffusion-reaction equations on distorted meshes. Computer Methods in Applied Mechanics and Engineering, 199(21):1386-1402, 2010.

[52] J. Principe, R. Codina, and F. Henke. The dissipative structure of variational multiscale methods for incompressible flows. Computer Methods in Applied Mechanics and Engineering, Accepted, 2009 . 
[53] Rogallo. Numerical experiments in homogeneous turbulence. Number 81315 in NASA Technical Memorandum. 1981.

[54] P. Sagaut. Large eddy simulation for incompressible flows. Scientific Computing, Springer, 2006.

[55] O. Schenk and K. Gärtner. Solving unsymmetric sparse systems of linear equations with PARDISO. Future Generation Computer Systems, 20(3):475 - 487, 2004.

[56] O. Schenk and K. Gärtner. On fast factorization pivoting methods for sparse symmetric indefinite systems. Electronic Transactions on Numerical Analysis, 23:158-179, 2006. 